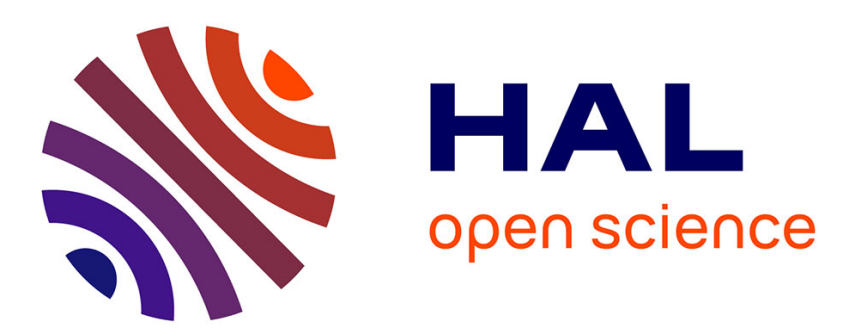

\title{
Evolution of the wall shear stresses during the progressive enlargement of symmetric abdominal aortic aneurysms
}

A.-V. Salsac, S.R. Sparks, Jean-Marc Chomaz, J.C. Lasheras

\section{- To cite this version:}

A.-V. Salsac, S.R. Sparks, Jean-Marc Chomaz, J.C. Lasheras. Evolution of the wall shear stresses during the progressive enlargement of symmetric abdominal aortic aneurysms. Journal of Fluid Mechanics, 2006, 560 (august), pp.19-51. 10.1017/s002211200600036x . hal-01023355

\section{HAL Id: hal-01023355}

https: / hal-polytechnique.archives-ouvertes.fr/hal-01023355

Submitted on 28 Jul 2014

HAL is a multi-disciplinary open access archive for the deposit and dissemination of scientific research documents, whether they are published or not. The documents may come from teaching and research institutions in France or abroad, or from public or private research centers.
L'archive ouverte pluridisciplinaire HAL, est destinée au dépôt et à la diffusion de documents scientifiques de niveau recherche, publiés ou non, émanant des établissements d'enseignement et de recherche français ou étrangers, des laboratoires publics ou privés. 


\title{
Evolution of the wall shear stresses during the progressive enlargement of symmetric abdominal aortic aneurysms
}

\author{
By A.-V. SALSAC ${ }^{1,2}$, S. R. SPARK $\mathrm{S}^{1,3}$, J.-M. CHOMAZ \\ AND J. C. LASHERAS ${ }^{1}$ \\ ${ }^{1}$ Department of Mechanical and Aerospace Engineering, University of California San Diego, \\ La Jolla, CA 92093, USA \\ ${ }^{2}$ LadHyX, CNRS-Ecole Polytechnique, 91128 Palaiseau Cedex, France \\ ${ }^{3}$ School of Medicine, University of California San Diego, La Jolla, CA 92093, USA
}

(Received 29 September 2004 and in revised form 12 December 2005)

The changes in the evolution of the spatial and temporal distribution of the wall shear stresses (WSS) and gradients of wall shear stresses (GWSS) at different stages of the enlargement of an abdominal aortic aneurysm (AAA) are important in understanding the aetiology and progression of this vascular disease since they affect the wall structural integrity, primarily via the changes induced on the shape, functions and metabolism of the endothelial cells. Particle image velocimetry (PIV) measurements were performed in in vitro aneurysm models, while changing their geometric parameters systematically. It has been shown that, even at the very early stages of the disease, i.e. increase in the diameter $\leqslant 50 \%$, the flow separates from the wall and a large vortex ring, usually followed by internal shear layers, is created. These lead to the generation of WSS that drastically differ in mean and fluctuating components from the healthy vessel. Inside the AAA, the mean WSS becomes negative along most of the aneurysmal wall and the magnitude of the WSS can be as low as $26 \%$ of the value in a healthy abdominal aorta.

Two regions with distinct patterns of WSS were identified inside the AAA: the proximal region of flow detachment, characterized by oscillatory WSS of very low mean, and the region of flow reattachment, located distally, where large, negative WSS and sustained GWSS are produced as a result of the impact of the vortex ring on the wall.

Comparison of the measured values of WSS and GWSS to an analytical solution, calculated for slowly expanding aneurysms shows a very good agreement, thus providing a validation of the PIV measurements.

\section{Introduction}

An abdominal aortic aneurysm (AAA) is a localized, permanent dilatation of the infrarenal abdominal aorta similar to a balloon. AAAs pose a health risk due to their potential for thrombus formation, dissection and more importantly, rupture. They are a significant and common vascular problem, whose rate of incidence has greatly increased with the increase in the life expectancy of the population (Best, Price \& Fowkes 2003). Diagnosis and treatment of this vascular disease has become a priority in health management of the elderly population. AAAs are often not detected at early stages and, in most cases, remain latent until symptoms occur as their size 
greatly increases or until they are diagnosed during an incidental exam (Szilagyi 1982). Their natural progression is that of gradual expansion. However, because of the lack of knowledge of the role that various factors play in the expansion process, no accurate technique exists to date to either predict the aneurysmal expansion rate or to determine its critical size (or shape) at the point of rupture. Although it is widely recognized that increase in size leads to a higher risk of rupture (Englund et al. 1998), small size AAAs may also rupture (Darling 1970), while others have been observed to grow to very large sizes without rupturing. For lack of any other reliable method, aneurysm diameter is the current standard by which physicians estimate the risk of rupture (Stringfellow, Lawrence \& Stringfellow 1987). Treatment is currently recommended for AAAs exceeding $5 \mathrm{~cm}$ in maximal diameter (Prisant \& Mondy 2004).

Although the mechanisms responsible for the AAA formation are not established, several risks factors have been identified such as age, gender, smoking, diabetes, high cholesterol and an unknown genetic component (Reilly \& Tilson 1989; Bengtsson, Sonesson \& Bergqvist 1996). The most common cause of AAAs was traditionally attributed to atherosclerosis, but recent evidence suggests that a more complex pathophysiology than atherosclerosis involving alterations in the levels of collagenase, elastase and antiprotease may contribute to the weakening of the aortic wall and to the enlargement of the AAA. Therefore the aetiology of AAAs has to be multifactorial and predominantly degenerative.

The prevailing hypothesis is that AAAs result from the coupling between structural changes in the intimal and medial layers of the arterial wall and disturbed patterns of haemodynamic stresses acting on the vessel wall. Any structural or conformational wall change may influence the flow in this arterial segment and downstream of it. Similarly, changes in the blood flow will result in altered pressure and wall shear stresses (WSS) and may lead to wall inflammation, thrombus formation, calcification and breakdown of the wall integrity (Fox \& Hugh 1966; Glagov et al. 1988; Bluestein et al. 1996; Pedersen et al. 1997). Thus, once an aneurysm is formed, it is reasonable to assume that expansion and rupture are not purely dependent on cellular and molecular processes, but rather on an interplay between mechanical stimuli exerted by the pulsatile blood flow and physiological wall changes. As detailed later in this section, past investigators have shown that the so-called 'disturbed' flow conditions that develop inside the AAA, such as regions of very high shear stresses, very low shear stresses, low but oscillating shear stresses and high wall shear stress gradients, may all contribute in various ways to the vascular disease, primarily via their effect on the endothelium.

The endothelium, a single layer of cells that line the blood vessels, plays a key role in the wall physiology because of its multiple regulatory functions: (i) the endothelial cells form a permeable barrier that prevents large molecules from entering the arterial wall; (ii) they detect vascular injury and produce substances to signal blood cells (leukocytes and platelets) to stick to the injured region and others to change the 'nature' of the vascular muscle cells causing them to migrate to the area of injury (Sterpetti et al. 1993; Emerson et al. 1999; Liu et al. 2003), and, more importantly, (iii) they act as a sensor/actuator to sense mechanical forces (pressure and shear stresses) exerted by the blood flow and release substances (nitric oxide, prostacyclin, etc), which modify the 'contractile tone' of the vascular muscle cells in the medial layer of the wall. As the principal recipient of haemodynamic forces (Dewey et al. 1981; Traub \& Berk 1998), the endothelial cells (EC) are the first to suffer from dysfunctions caused by the disturbed flow conditions that develop inside the AAA. 
The physiological response of cells to haemodynamic stresses, also called 'mechanotransduction', has been extensively investigated owing to the persistent rise of cardiovascular diseases over the last 10 years (Chen et al. 1999; Tzima et al. 2002; Zhao et al. 2002). Shear stress influences the baseline set point of dozens of physiologically important genes via transcription regulation (Chien, Li \& Shyy 1998). It also affects endothelial secretion of prostacyclin, a vasodilator and a potent inhibitor of platelet aggregation and of nitric oxide, which is thought to reduce leukocyte adhesion (Qiu \& Tarbell 2000). Endothelial cell damage, or even loss, have been reported in areas of high wall shear stresses, which are responsible for changes in the cell metabolism and for an increased permeability (Fry 1968). Endothelial cell expression has also been found to be upregulated in regions of low or oscillatory wall shear stress $(\mathrm{Ku}$ et al. 1985). Owing to the lack of mechanical stimuli, the cells become more round in shape, increasing the intra-cellular space and thus the wall permeability to macromolecules, such as low-density lipoproteins (LDL).

The effect of gradients of wall shear stresses (GWSS) has been studied only recently. They appear to induce an increased cell division rate and a net cell migration away from these regions (DePaola et al. 1992; Davies, Mundel \& Barbee 1995; Tardy et al. 1997; Nagel et al. 1999). Davies et al. (1986) showed that, under turbulent shear stresses, the endothelial cells suffered from an increased turnover along with changes in their shape and metabolism, and the cells failed to align with the flow. Therefore, in order to understand clinically the degree of endothelial dysfunction and its contributions to the aetiology and progression of the vascular disease, it is imperative to first quantify the evolution of the spatial and temporal distribution of the wall shear stresses resulting from the changes in geometry occurring during the enlargement of the aneurysm.

Owing to the complexity of measuring the flow inside an aneurysm, there has been no successful attempt at measuring the WSS in vivo inside an abdominal aortic aneurysm. None of the current radiological imaging techniques (MRI, ultrasound, etc.) yet has a high enough spatial resolution to provide a reliable measurement of the velocity field, let alone of the wall shear stresses.

Consequently, over the last decade, a fairly large number of studies have been conducted using in vitro models to investigate the haemodynamics in AAAs. These studies have been performed in both symmetric and non-symmetric idealized-shape models of AAA. Many of the studies involve steady flows (Schrader et al. 1992; Budwig et al. 1993; Peattie et al. 1994; Asbury et al. 1995; etc), which is not relevant to the problem at hand, since the flow is highly pulsatile in the aorta. Others (Yu et al. 1999; Yu 2000; Yu \& Zhao 2000) measured the velocity field in AAAs for unsteady flows, but used a sinusoidal waveform instead of a physiologically correct waveform, which strongly affects the characteristics of the flow.

Fukushima, Matsuzawa \& Homma (1989) were the first to investigate the pulsatile nature of the flow, reproducing the physiologically correct velocity waveform. They studied experimentally the influence of the geometry of the bulge in three axisymmetric models of AAA, in the range of mean Reynolds numbers $289 \leqslant\langle\bar{R} e\rangle \leqslant 748$ and Womersley number $4.07 \leqslant \alpha \leqslant 10.6$. They showed that the flow remained attached to the walls during the acceleration (systole), but detached at the onset of the deceleration (beginning of diastole), generating a large primary vortex, followed by a weaker secondary vortex, and a recirculation zone dominated by very low velocities. Although this study showed qualitatively the most significant haemodynamic changes occurring as a result of the bulging, it did not provide any measurement of the most physiologically relevant parameter, i.e. the wall shear stresses. Instead, a numerical 
simulation of the idealized laminar flow inside the AAA was realized and showed that, with the exception of the distal area, where the WSS peaked to a value of $1.5 \mathrm{~Pa}$, the aneurysm wall was characterized by low WSS.

A similar qualitative study was conducted by Egelhoff et al. (1999) in four symmetric and one non-symmetric models of AAAs but they did not perform measurements of the WSS either. Measuring the WSS experimentally is challenging, since it requires a precise measurement of the velocity at points very close to the wall, in order to assess the velocity gradient at the wall. Yip \& Yu (2001) published one set of laser doppler anemometry measurements of WSS. However, they could not measure the spatial distribution of WSS, which is believed to be one of the most important factors leading to physiological changes.

A few groups performed numerical studies of the velocity field and WSS in AAAs. Some studies reproduced non-realistic geometries, such as Viswanath, Rodkiewicz \& Zajac (1997) who modelled extremely large AAAs $(6 \mathrm{~cm} \leqslant D \leqslant 14 \mathrm{~cm})$ or Finol \& Amon (2001) and Finol \& Amon $(2002 a, b)$ who modelled a double aneurysm, made out of two consecutive expansions. Numerical calculations have difficulty in correctly predicting flow separation as well as the transition to turbulence, which are the two important characteristics of the aneurysmal flow.

All the above studies, both experimental and numerical, provide a good qualitative description of the flow in an AAA. However, no comprehensive quantitative study of the evolution of the flow field as the AAA enlarges has been reported and, more importantly, there has been no accurate measurements of the evolution of the WSS. The aim of our study is, therefore, to conduct precise measurements of the spatial and temporal changes in wall shear stresses acting on the endothelial cells as the AAA grows. In the following, we will discuss the results of a parametric study, in which the flow characteristics were studied inside the AAA, while varying the geometric parameters of the models systematically. The models considered in this study are all rigid and symmetric in shape. Quantitative measurements of the velocity field inside the AAA models are obtained using particle image velocimetry (PIV) while reproducing a physiologically correct pulsatile flow waveform. The haemodynamic stresses acting on the vessel wall are then calculated in each model from the measured velocity field. In addition, in order to validate our experimental measurements of the WSS, we analyse these measurements in the context of an analytical model of the flow inside a healthy aorta as well as in an AAA, which was developed based on the wellknown Womersley solution.

A description of the experimental set-up and method is given in $\S 2$. Section 3 describes the flow in a healthy infrarenal aorta measured experimentally. The results are compared to the standard analytical results of a pulsatile flow in a straight infinitely long tube. Section 4 details the results of the parametric study performed in in vitro models of AAAs and of the analytical solution. A discussion about the physiological implications of the measured changes in flow properties resulting from the AAA enlargement is given in $\S 5$.

\section{Experimental set-up}

The experiments consist of a parametric study in symmetric models of AAA. The models tested in the experiment are made of a fusiform expansion, blown in a straight glass tube as shown in figure 1 . The models do not preserve the physiologically correct geometry, but they still retain all the important physical processes that occur in an AAA. Flow separation, formation of a strong vortex followed by an internal shear 


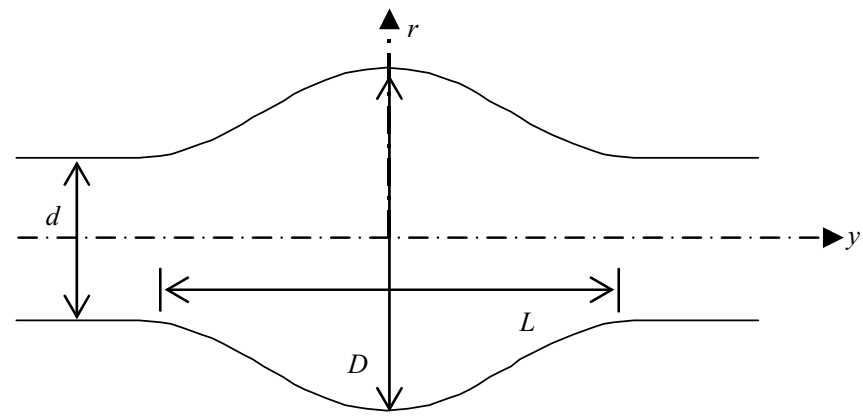

FIGURE 1. Geometry of the AAA models.

$D / d \quad \overbrace{2.9} \begin{array}{cccc}1.3 & 3.9 & 5.2 \\ 1.6 & 1 & 6 & 11 \\ 1.9 & 2 & 7 & 12 \\ 2.1 & 3 & 8 & 13 \\ 2.4 & 4 & 9 & 14 \\ & 5 & 10 & 15\end{array}$

TABLE 1. Geometrical characteristics of the different aneurysm models tested experimentally.

layer, impingement of the jet, and transition to turbulence are all observed in the simplified models of AAA, along with regions of high/low WSS and high GWSS. The advantage is that these well-controlled geometrical shapes are characterized by only two geometric parameters: the aspect ratio $L / d$ and the dilatation ratio $D / d$, where $D$ and $L$ are, respectively, the maximum inner diameter and length of the aneurysm bulge and $d$ is the inner diameter of the parent vessel. These parameters have been systematically varied in the study in order to analyse the changes in the haemodynamic forces as the aneurysm enlarges (see table 1). All of the models selected for our study correspond to limited size aneurysms $(D<4.5 \mathrm{~cm})$, which are clinically shown to be typically devoid of an endoluminal thrombus. This would not hold for larger aneurysms, in which a thrombus has been observed in $75 \%$ of the cases (Harter et al. 1982).

The aneurysm models are rigid ones made out of glass. Even in the case of intracranial aneurysms, Steiger et al. (1987) and Duncan et al. (1990) showed that the vessel compliance only slightly modifies the magnitude of the shear stresses and does not change the general characteristics of the flow. Furthermore, unlike intracranial aneurysms, AAAs actually become stiffer as they expand so that their wall compliance become nearly negligible compared to a healthy aorta (Di Martino et al. 1998). In vivo, wall compliance still plays an important role in the shaping of the pressure waveform generated by the heart, as the pressure pulse travels along the vasculature. However, in our experiments, the flow input into the model is not the cardiac flow waveform, but the one measured in vivo in the infrarenal aorta. Thus, the rigidity of the models does not impair the flow waveform.

Figure 2 schematically shows the experimental flow facility. The pulsatile flow is provided by a programmable piston pump. A rack-mounted piston is driven in a cylinder by a computer-controlled micro-stepping motor (Compumotor Corporation, 


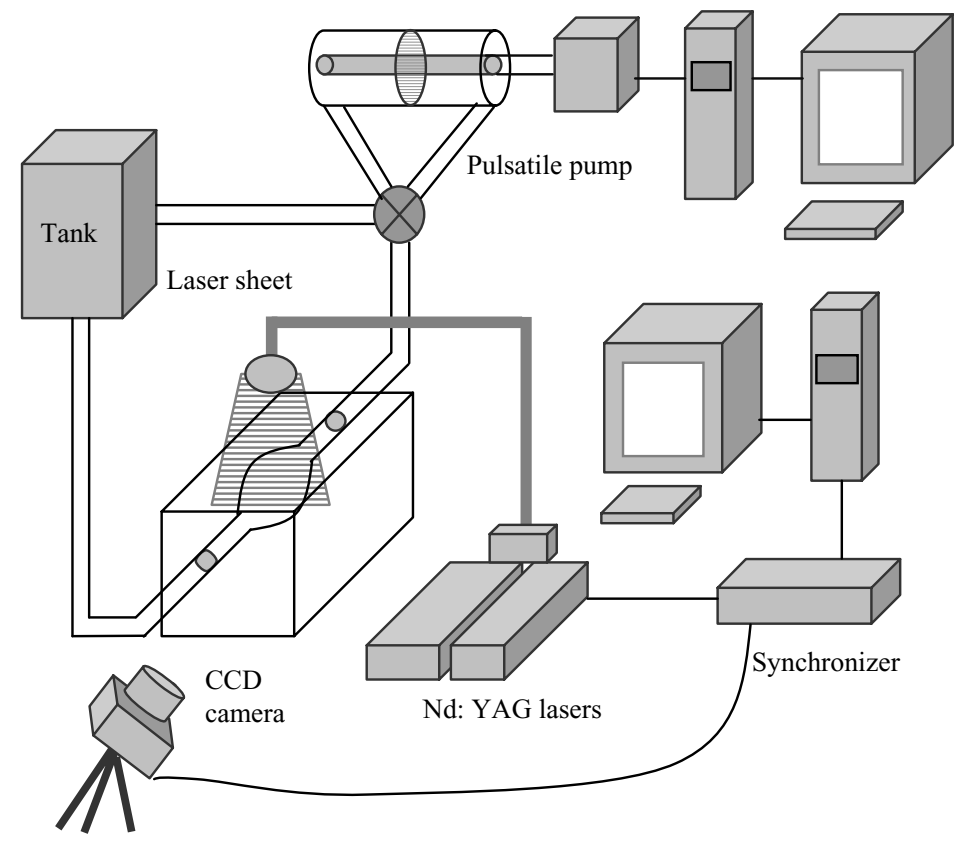

FIGURE 2. Experimental set-up.

Cupertino, CA). The piston divides the cylinder into two parts. A four-way pool valve (Numatics, Highlands, MI) interchanges the inlet and outlet pathways each time the piston reaches the end of its journey. The valve allows the pump to continuously empty one side of the cylinder and refill the other, while keeping the same flow direction in the test section. The pump is programmed with flow patterns corresponding to the aortic flow measured in the infrarenal aorta in a healthy adult individual. The physiological flow waveform employed in this study is based on the results reported by Maier et al. (1989) for a patient at rest. The available torque of the stepper motor $(0.5 \mathrm{Nm})$ and the cylinder volume $(0.4501)$ constituted a limit for the experiment. Since the highest flow rate delivered by the pump was smaller than the physiological subrenal aortic flow, the whole experiment was scaled down in size by a geometric factor of approximately $2(d=9 \mathrm{~mm}$ in the experiment) and pure water was used, water being approximately four times less viscous than whole blood. Pulsated Newtonian fluids are fully characterized by two non-dimensional parameters. Complete similarity is achieved by reducing the velocity by a factor of 2 and increasing the frequency by $15 \%$. The Reynolds number, $R e=U d / v$, ratio of the inertial forces to the viscous forces, and the Womersley number, $\alpha=d / 2 \sqrt{\omega / \nu}$, ratio of the unsteady inertial forces to the viscous forces, were kept identical to the infrarenal flow (where $U$ is the characteristic velocity, $v$ the kinematic viscosity of the fluid, $\omega=2 \pi f$ the pulsation of the flow, and $f$ the frequency). All the physical quantities (velocity, vorticity, stress, WSS, GWSS, etc.) presented in this paper have been converted into the physiologically correct ones.

By using water as the perfusion fluid, we assumed blood to behave as a Newtonian fluid for the application of interest. Attinger (1964) and El-Khatib \& Damiano (2003) among others showed that, in large-sized vessels, the non-Newtonian behaviour of blood was limited to minor deviations confined to the vessel core where the shear stresses are low. Since the primary aim of our study is to measure the stresses close to the wall, where they are maximum, the Newtonian approximation should still provide 
accurate measurements of the WSS. Furthermore, with a diameter around 16-21 mm, the abdominal aorta is much larger than the critical value of $1 \mathrm{~mm}$ measured by Nichols \& O'Rourke (1990), below which non-Newtonian effects cannot be neglected.

The instantaneous velocity field inside the AAA is measured using a twodimensional PIV system (TSI Incorporated, St Paul, MN). This system is composed of two $50 \mathrm{~mJ}$ pulsed Nd:YAG lasers, a synchronizer and a CCD camera (figure 2). The lasers fire at a frequency of $10 \mathrm{~Hz}$, with a time interval of 5-10 ns between each of them, thus providing sufficient temporal resolution to characterize the flow in a complete cardiac cycle. The laser beams are spread into a light sheet by an array of spherical and cylindrical lenses. The thickness of the sheet is about $0.5 \mathrm{~mm}$ at the focal point. The flow is seeded with $10 \mu \mathrm{m}$-diameter lycopodium particles (Carolina Biological Supply Company, Burlington, NC). The AAA models are placed in a transparent box filled with water in order to limit optical deformations due to refraction. In the case of transverse measurements, the use of a prism in contact with the free surface of the water contained in the box enabled us to avoid any problem resulting from the free surface distortion and changes in the index of refraction (see Schowalter, Van Atta \& Lasheras 1994 for further information).

A 630046 PIVCAM 10-30 camera, with a resolution of $1024 \times 1024$ pixels and an 8-bit dynamic range, is synchronized with the lasers and records the position of the light-scattering particles in the illuminated plane. These digital image pairs are processed using the software Insight, which calculates the instantaneous velocity field in the measuring plane. The cross-correlation function is computed using twodimensional fast Fourier transforms. Its peak, calculated at a subpixel precision, is located using a fit with a two-dimensional Gaussian of adjustable width.

\section{Characteristics of the flow in a healthy abdominal aorta}

\subsection{Haemodynamics in a healthy abdominal aorta}

\section{Experimental results}

Before discussing the flow characteristics in an aneurysmal aorta, we shall first characterize the flow in a healthy infrarenal aorta as the reference case. The infrarenal aorta is idealized as a straight pipe of diameter $d$. The model is supplied with the typical pulsatile inflow waveform measured in a male subject at rest. The mean flow rate is $11 \mathrm{~min}^{-1}$ and the simulated heart rate 71 pulses $\mathrm{min}^{-1}$. This input flow condition corresponds to a peak Reynolds number $\bar{R} e_{p}=2700$, a time-averaged Reynolds number $\langle\bar{R} e\rangle=330$ and a Womersley number $\alpha=10.7$, where the Reynolds numbers are calculated based on the flow rate. The input waveform of the flow rate, non-dimensionalized by the systolic peak, is plotted in figure 3(a).

The time evolution of the velocity field was measured in the central axial plane of the tube using the PIV system. The measurements were taken zooming on only half the vessel in order to achieve a high spatial resolution (mesh size $0.019 d \times 0.019 d$ ). Figure $3(b)$ shows, at a few instants of time in the cardiac cycle, the profiles of the longitudinal velocity, $v$, phase-averaged over 6 cardiac cycles. At the beginning of the cycle, the fluid is almost at rest (figure $3 b \mathrm{~A}$ ). During the acceleration portion of the systole (time B), the flow develops into the characteristic top-hat velocity profile. When the Womersley number is small, viscous forces dominate and the velocity profiles are parabolic in shape. However, for a Womersley number above 10, which is the case in the abdominal aorta, the unsteady inertial forces dominate, and the flow is nearly top hat with thin boundary layers. At the peak systole, the thickness 
(a)

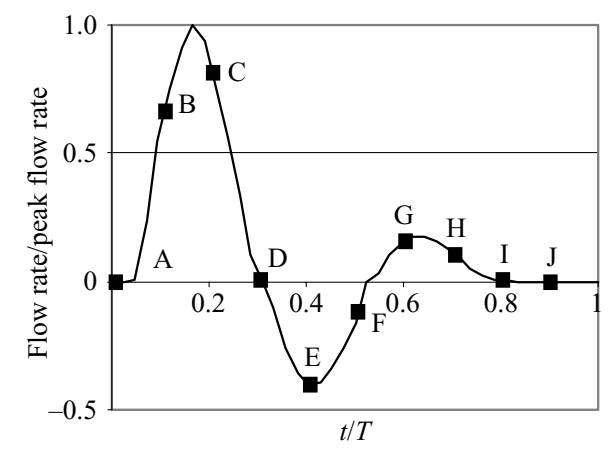

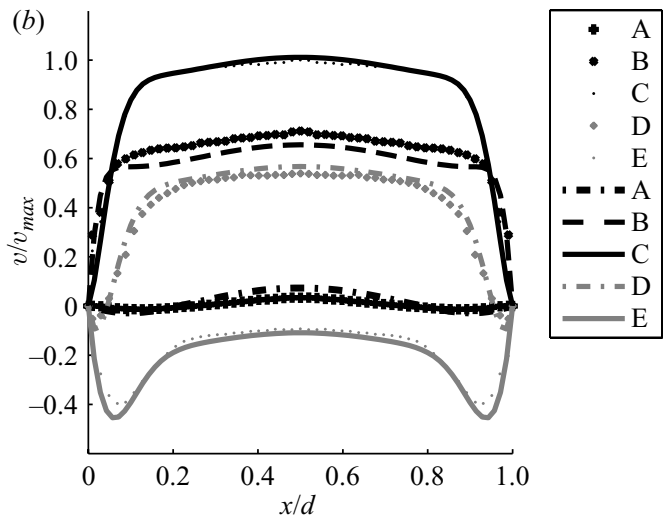

FIGURE 3. (a) Flow waveform input in the pump, non-dimensionalized by the peak flow rate $Q_{p}$. (b) Velocity profiles measured across the healthy abdominal aorta at several instants of time in one cardiac cycle, non-dimensionalized by the velocity based on $Q_{p}$ (single points). Comparison with the velocity profiles calculated with the Womersley solution (dotted curves). The $y$-axis is along the symmetry axis of the model and the $x$-axis is in the transverse direction.

of the boundary layer scales as $d / \alpha$. After the peak systole, the flow decelerates first along the walls and quickly reverses, while the bulk of the fluid still moves forward, with a blunt velocity profile (time D). The bulk of the flow reverses only at peak diastole (times E-F). At the end of the diastole, the flow sets forward again (times G-H) and over the resting period (times I-J), the flow relaxes to near rest before being accelerated again at the beginning of the next cardiac cycle. It is important to point out that although the flow develops an inflectional velocity profile during diastole, it remains entirely laminar during the whole cardiac cycle. At the high values of Womersley number corresponding to these measurements, the characteristic time for the growth of the instability is much longer than the period of the pulsatility.

\section{Analytical solution}

The PIV measurements in a healthy vessel can be compared to what is commonly called the 'Womersley solution'. As a first approximation, the healthy abdominal aorta can be modelled as a straight infinite rigid tube. The flow of a viscous fluid in an infinite tube under a periodic pressure gradient was first studied by Richardson \& Tyler (1929). Womersley (1954) and Helpes \& McDonald (1954) calculated analytical solutions for the arterial pulsating flow expressing the time-varying pressure gradient as the Fourier series of sinusoidal modes (see also Pedley 1979).

The longitudinal velocity $v(r, t)$, non-dimensionalized by a characteristic velocity $U$, can be expressed as

$$
v^{*}\left(r^{*}, t^{*}\right)=\frac{R e G_{0}^{*}}{4}\left(1-r^{* 2}\right)+\frac{R e}{\mathrm{i}} \sum_{n=1}^{\infty} \frac{G_{n}^{*}}{\alpha_{n}^{2}}\left(1-\frac{J_{0}\left(r^{*} \mathrm{i}^{3 / 2} \alpha_{n}\right)}{J_{0}\left(\mathrm{i}^{3 / 2} \alpha_{n}\right)}\right) \mathrm{e}^{\mathrm{in} t t^{*}},
$$

where the lengths are non-dimensionalized by the radius $a_{0}=d / 2$ and the time by the pulsation frequency $\omega$ (the stars indicate the dimensionless variables). $J_{0}$ is the Bessel function of the first kind of order 0 and $\alpha_{n}=a_{0} \sqrt{n \omega / v}$. One can remark that $\alpha_{1}=a_{0} \sqrt{\omega / \nu}$ is the Womersley number $\alpha$ defined in $\S 2$. $G_{n}^{*}$ are the Fourier coefficients of the pressure gradient, where the pressure is non-dimensionalized by $\rho U^{2}$. In order to compute the velocity field induced by the pump in the experiment, the pressure coefficients can be calculated from the Fourier decomposition of the input flow rate 
(of Fourier coefficients $Q_{n}^{*}$ )

$$
G_{0}^{*}=\frac{8}{\pi R e} Q_{0}^{*} ; G_{n}^{*}=\frac{\mathrm{i} \alpha_{n} * 2}{\operatorname{Re}\left(1-F\left(\alpha_{n}\right)\right)} Q_{n}^{*},
$$

where

$$
F\left(\alpha_{n}\right)=\frac{2}{\mathrm{i}^{3 / 2} \alpha_{n}} \frac{J_{1}\left(\mathrm{i}^{3 / 2} \alpha_{n}\right)}{J_{0}\left(\mathrm{i}^{3 / 2} \alpha_{n}\right)} .
$$

Figure $3(b)$ compares the measured velocity profile to those calculated with the Womersley solution. The difference between the experimental and the theoretical profiles is found to be about $8 \%$, whereas the standard deviation between the 6 independent realizations that have been used to compute the average is $3.7 \%$. The agreement between the measured velocity field and the theoretical laminar profile confirms the standard result that although the velocity profile is highly inflectional after the peak systole, the unsteadiness of the flow prevents the instability from developing. The small differences between the measured and the theoretical profiles may be attributed to a small variation (of a few per cent) of the input wave profile from the pump with the effective waveform that enters the model. This could be due either to an inaccuracy of the pump or to modifications of the waveform between the outlet of the pump and the model owing to entrance effects or to a weak pressure peak damping. However, it is important to notice, in figure $3(b)$, that the first two measurement points closest to the wall are placed almost on the theoretical curve at each time step. This should guarantee a good measurement of the wall shear stress.

\subsection{Wall shear stresses in a healthy abdominal aorta}

\section{Experimental results}

The evolution of the wall shear stresses was calculated over time from the velocity measurements described above. In the case of a laminar axisymmetric flow, the WSS is simply given by

$$
W S S=-\mu \frac{\partial v}{\partial x} .
$$

The measured temporal evolution of wall shear stress, plotted in figure 4, follows the evolution of the flow rate. The WSS ranges from $-3 \mathrm{~Pa}$ to $4.9 \mathrm{~Pa}$, the extrema occurring, respectively, at peak diastole and peak systole. The convention is to assign a negative value to WSS corresponding to reversed flow. As mentioned in $\$ 2$, all the measured values correspond to the physiologically correct flow. The time-averaged WSS,

$$
W S S_{\text {mean }}=\frac{1}{T} \int_{0}^{T} W S S \mathrm{~d} t
$$

is measured as equal to $0.27 \mathrm{~Pa}, T$ being the period of the cardiac cycle. The timeaverage of the magnitude of the shear stress,

$$
W S S_{m a g}=\frac{1}{T} \int_{0}^{T}|W S S| \mathrm{d} t,
$$

is equal to $1.5 \mathrm{~Pa}$. The oscillating shear index,

$$
O S I=\frac{1}{2}\left(1-\frac{W S S_{\text {mean }}}{W S S_{\text {mag }}}\right)
$$




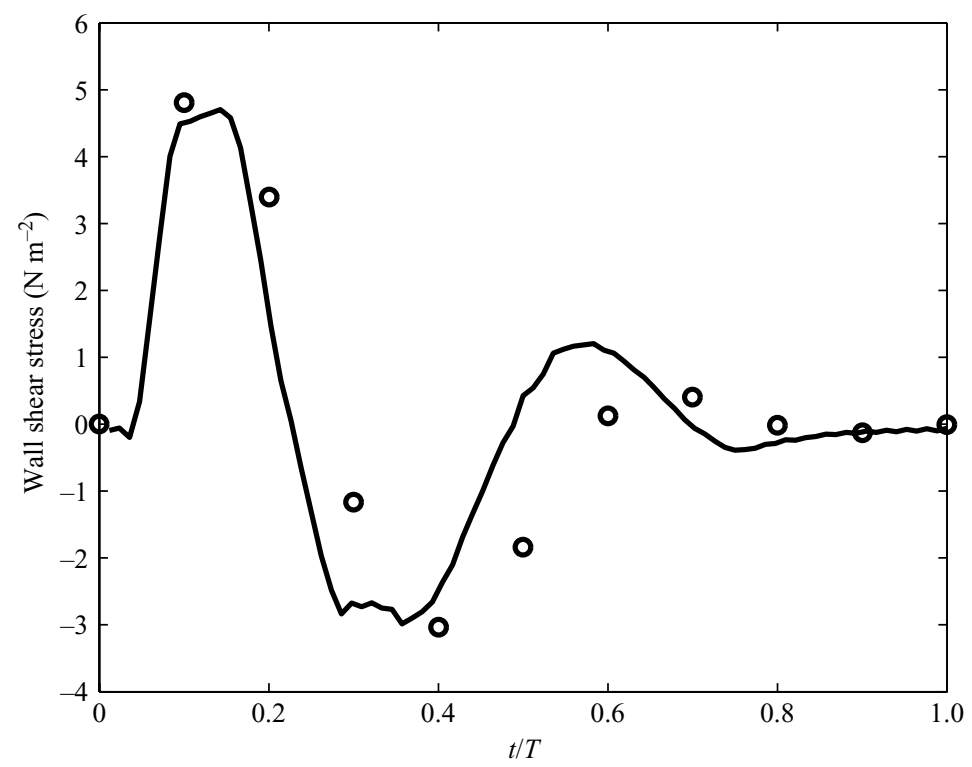

FIGURE 4. Comparison between the measured and calculated values of the wall shear stresses in a healthy infrarenal aorta.

is equal to 0.4 . This index quantifies the pulsatility of the flow and the main direction of the flow. It ranges from 0 (forward flow throughout the cardiac cycle) to 1 (fully reversed flow). An OSI index of 0.5 corresponds to a pure oscillating flow with a $W S S_{\text {mean }}$ of 0 .

Oyre et al. (1997) measured the wall shear stresses along the suprarenal and infrarenal aorta in vivo and non-invasively using magnetic resonance velocity mapping. They found WSS of comparable magnitude to our experiments $(-1.3 \mathrm{~Pa} \leqslant W S S \leqslant$ 4.9 Pa). From their curve of WSS, we can calculate an OSI index of 0.32 . Cheng, Parker \& Taylor (2002) and Taylor et al. (2002) made similar measurements with cine phase-contrast magnetic resonance imaging. However, in the case of a patient at rest, they only measured a peak wall shear stress of $2 \mathrm{~Pa}$ and a time-average of $0.16 \mathrm{~Pa}$. Their lower WSS values may be accounted for by the low spatial resolution of their measurements, which only allowed them to measure the velocity at 7 locations across the vessel. A higher resolution is required to obtain a good estimate of the gradient of the velocity at the wall.

\section{Analytical solution}

The wall shear stress, calculated from the velocity field (equation (3.1)),

$$
\operatorname{WSS}^{*}\left(t^{*}\right)=-\frac{\partial v^{*}}{\partial r^{*}} \|_{r^{*}=1}=\frac{\operatorname{Re} G_{0}^{*}}{2}+\sum_{n=1}^{\infty} \frac{\operatorname{Re} G_{n}^{*}}{2} F\left(\alpha_{n}\right) \mathrm{e}^{\mathrm{i} n t^{*}},
$$

predicts a peak WSS of $4.87 \mathrm{~Pa}, W S S_{\text {mean }}=0.16 \mathrm{~Pa}, W S S_{\text {mag }}=1.3 \mathrm{~Pa}$ and $O S I=0.42$. The measured values are therefore in good agreement with the analytical solution, apart from the mean value that is too low. This is due to the lower values of WSS during the resting period of the cardiac cycle. We can see that, experimentally, the WSS profile is smoother and does not increase back to a secondary peak during the resting period. It looks as if the pump and the experimental system in general were filtering the high-frequency modes. 
The WSS is the parameter that is physiologically relevant at the level of the endothelial cell response. This section showed that the WSS were fluctuating in a healthy abdominal aorta, where the OSI parameter can be between 0.32 and 0.42 . As discussed in $\S 1$, any departure from the healthy pattern of WSS strongly affects the morphology, metabolism and gene expression of the endothelial cells. In the next section, we will analyse how the presence of an AAA influences the spatial and temporal distribution of WSS.

\section{Flow in abdominal aortic aneurysms}

4.1. Results of the parametric study of the flow characteristics in $A A A$ Typical flow in an aneurysm

Before analysing the effect of aneurysmal growth on the flow topology, we will discuss the important spatial and temporal features, which characterize the typical flow field in an abdominal aortic aneurysm. For that purpose, we have selected an aneurysm with a dilatation ratio $D / d=2.1$ and an aspect ratio $L / d=2.9$ (model 4). The velocity field was measured in a central axial plane of the aneurysm with the PIV system with a mesh size of $0.068 d \times 0.068 d$. The vorticity and total stresses were calculated from the velocity field. The total stress is defined as the maximum eigenvalue of the stress tensor (see Appendix) and is therefore a good measure of the maximum strain rate developing in the flow. Figure 5 shows the instantaneous velocity field and figure 6 the corresponding vorticity and stress fields at times B to E. The same fields are shown in figure 7, phase-averaged over 6 cardiac cycles. The 10 measurements composing each sequence are equally spaced in time, and represent a full cardiac cycle. Time progresses with the sequential numbering of the frames as shown on the velocity waveform in figure $5 \mathrm{~K}$. Analysis of these measurements reveals important features of the haemodynamics in fusiform aneurysms, which are typical characteristics of the behaviour of pulsatile flows in a cavity. The striking feature is that the flow inside the aneurysm is dominated by the formation of separated flow regions with internal shear layer composed of an array of coherent vortex rings that traverse along the length of the AAA.

During the acceleration portion of the systole, the flow remains laminar and fully attached to the bulging walls (figures 5 and $7 a \mathrm{~A}-\mathrm{B}$ ). The fact that the flow remains attached in the divergent portion is a consequence of the positive pressure gradient generated at the beginning of the systole: the temporal acceleration of the flow is at this point larger than the convective deceleration originated by the upstream (proximal) diverging walls of the artery. Figure $6 \mathrm{~B}$ shows that, during the entire acceleration portion of the cardiac cycle, the vorticity and stresses are confined to very thin boundary layers and the bulk of the flow behaves as a potential flow (irrotational and inviscid).

Just after the initiation of the flow deceleration (figures 5 and $7 a \mathrm{C}$ ), the flow reverses close to the wall, while the bulk of the flow is still moving forward. At this point, the flow massively detaches from the proximal neck. A recirculating flow region forms in the proximal half of the aneurysm, while the flow remains attached to the wall further downstream. The vorticity field (figures $6 a$ and $7 b \mathrm{C}$ ), shows the roll up of the boundary layer into a very large start-up vortex. This intense vortex is followed by a free shear layer, in which some secondary vortices form owing to the Kevin Helmholtz instability. The effect of flow separation is also to displace the peak of shear stresses from the wall into the bulk of the flow (figures $6 b$ and $7 c \mathrm{C}$ ). 

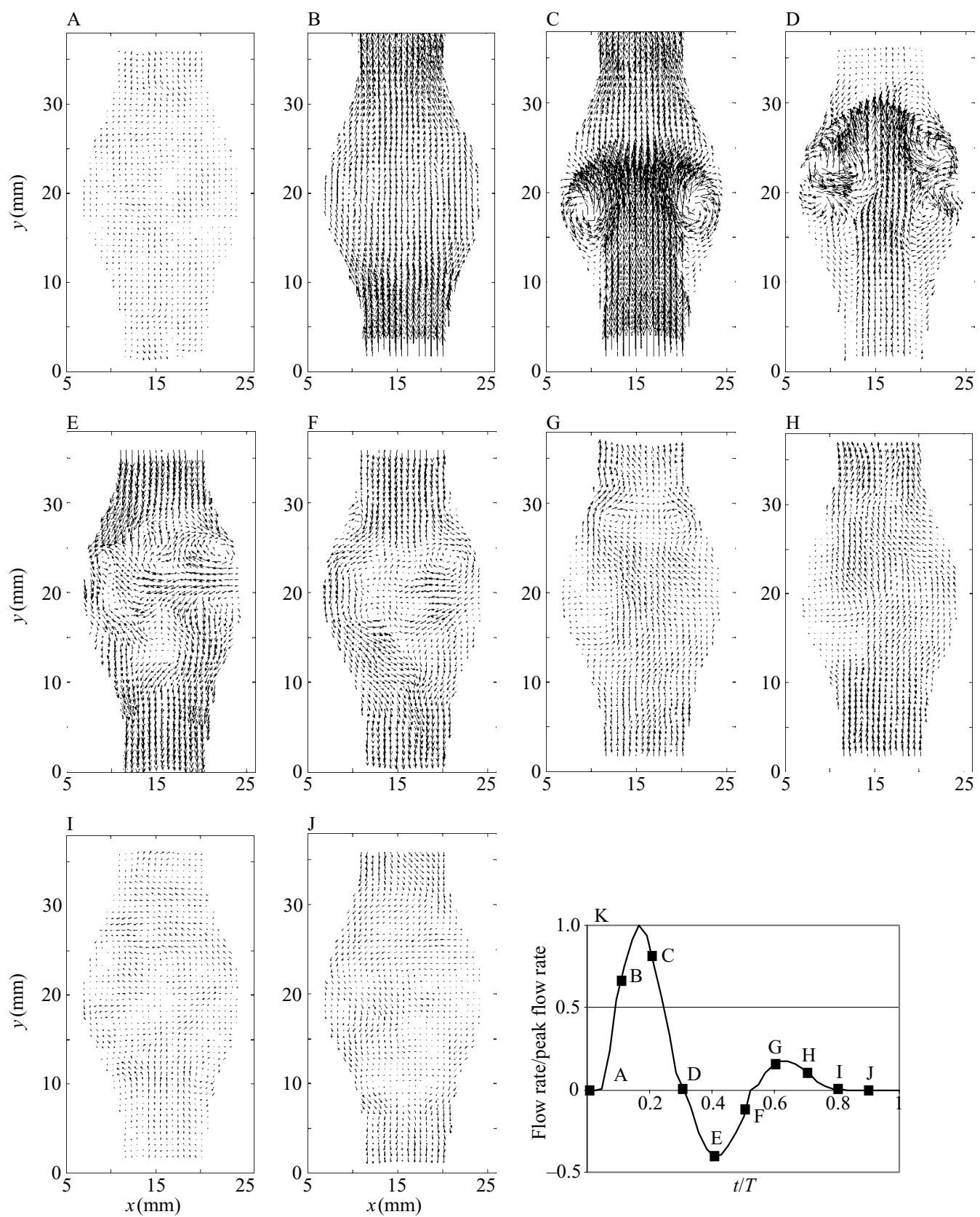

FIGURE 5. Instantaneous velocity field measured in model 4 with the PIV system.

The vortex ring travels along the aneurysm until it impinges on the distal neck of the AAA (figures 5 and $7 a \mathrm{D}$ ), resulting in a sharp increase in shear stresses in the converging portion of the AAA. At this point, the cylindrical internal shear layers span across the entire length of the AAA (figure $6 a \mathrm{D}$ ) and the flow is fully detached from the wall along the entire AAA length. At the point of impact, the boundary layer is characterized by vorticity and stresses of the opposite sign, because of the presence of the vortex ring. 

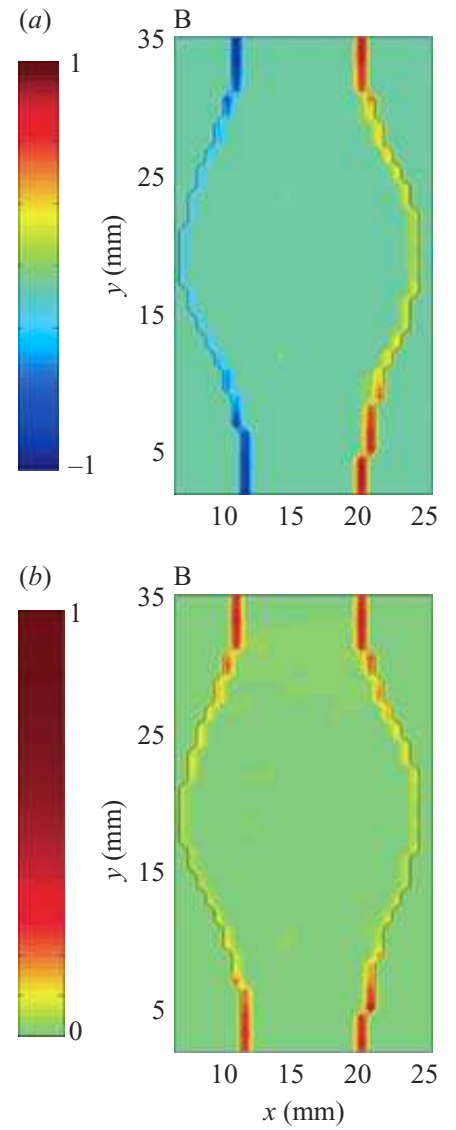
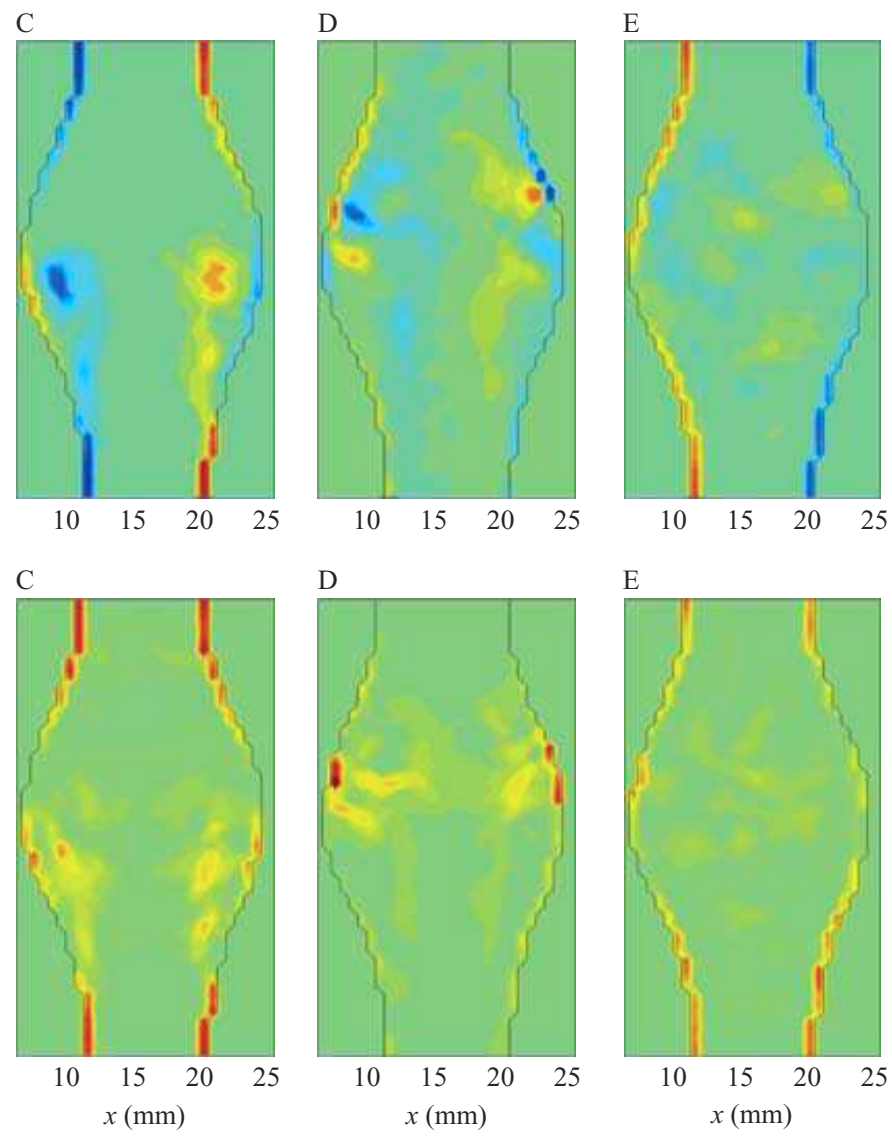

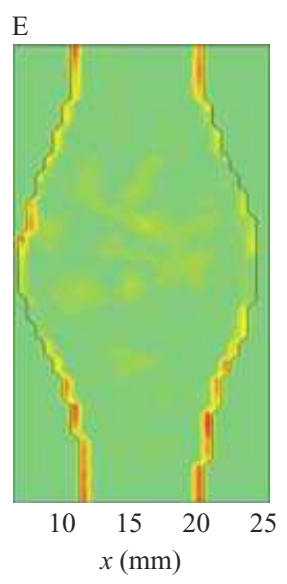

Figure 6. (a) Instantaneous vorticity and $(b)$ stress fields measured in model 4 at times B to $\mathrm{E}$, non-dimensionalized by the peak value occurring in the healthy vessel $\left(610 \mathrm{~s}^{-1}\right.$ for the vorticity and $2.45 \mathrm{~N} \mathrm{~m}^{-2}$ for the stress).

The characteristic length scale of the boundary layer becomes so small that the resolution is not sufficient to provide a reliable measurement of the flow characteristics very close to the wall at time $\mathrm{D}$. Therefore, we measured the flow field in model 4 , zooming in on the region near the distal wall, in order to increase the resolution (mesh size $0.027 d \times 0.027 d$ ). Figure 8 shows the velocity, vorticity and stress fields at time $\mathrm{D}$. The measurements show the primary vortex ring hitting the distal wall. On the vorticity plot (figure $8 b$ ), we can observe the change in sign of vorticity as we move from the centreline to the wall passing through the vortex ring. The presence of high velocity so close to the wall induces very thin boundary layers $(\sim 0.02 d)$ with stresses almost three times higher than in a healthy abdominal aorta $\left(\geqslant 6 \mathrm{~N} \mathrm{~m}^{-2}\right)$. Unfortunately, the resolution is still not high enough to resolve the boundary layer, resulting in a slightly underestimated measurement of the wall shear stresses.

A loss of the two-fold symmetry can be observed on the instantaneous fields as the vortex hits the wall. It corresponds to a loss in axisymmetry, which increases after the vortex ring has hit the distal wall (times $\mathrm{E}$ and $\mathrm{F}$ ). The resulting flow presents a larger randomness, which can be seen when comparing the instantaneous and phaseaveraged fields (figures 5-7). As the flow reverses in the diastole, the coherent shear layer and the preceding vortex ring break down, leading to disordered vortices of 
(a)

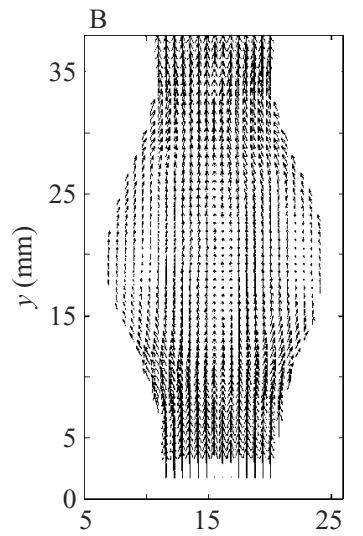

(b)
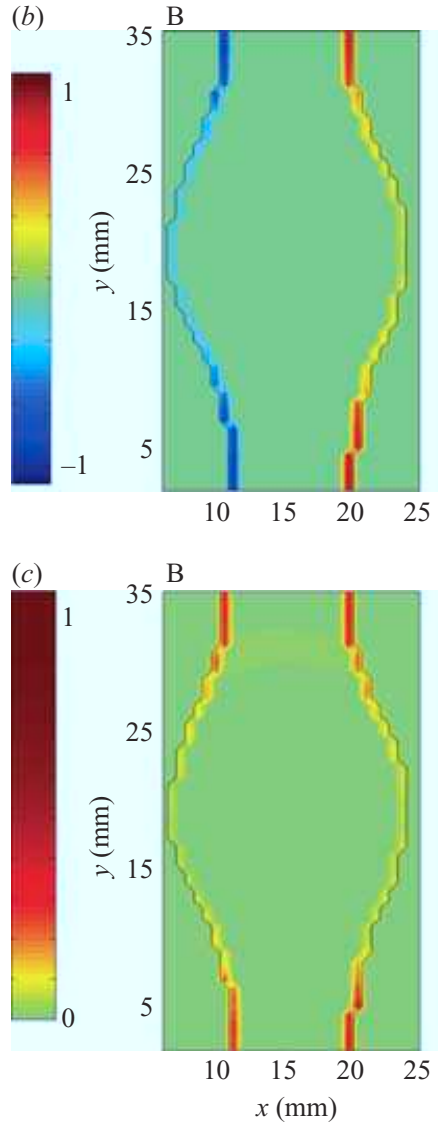
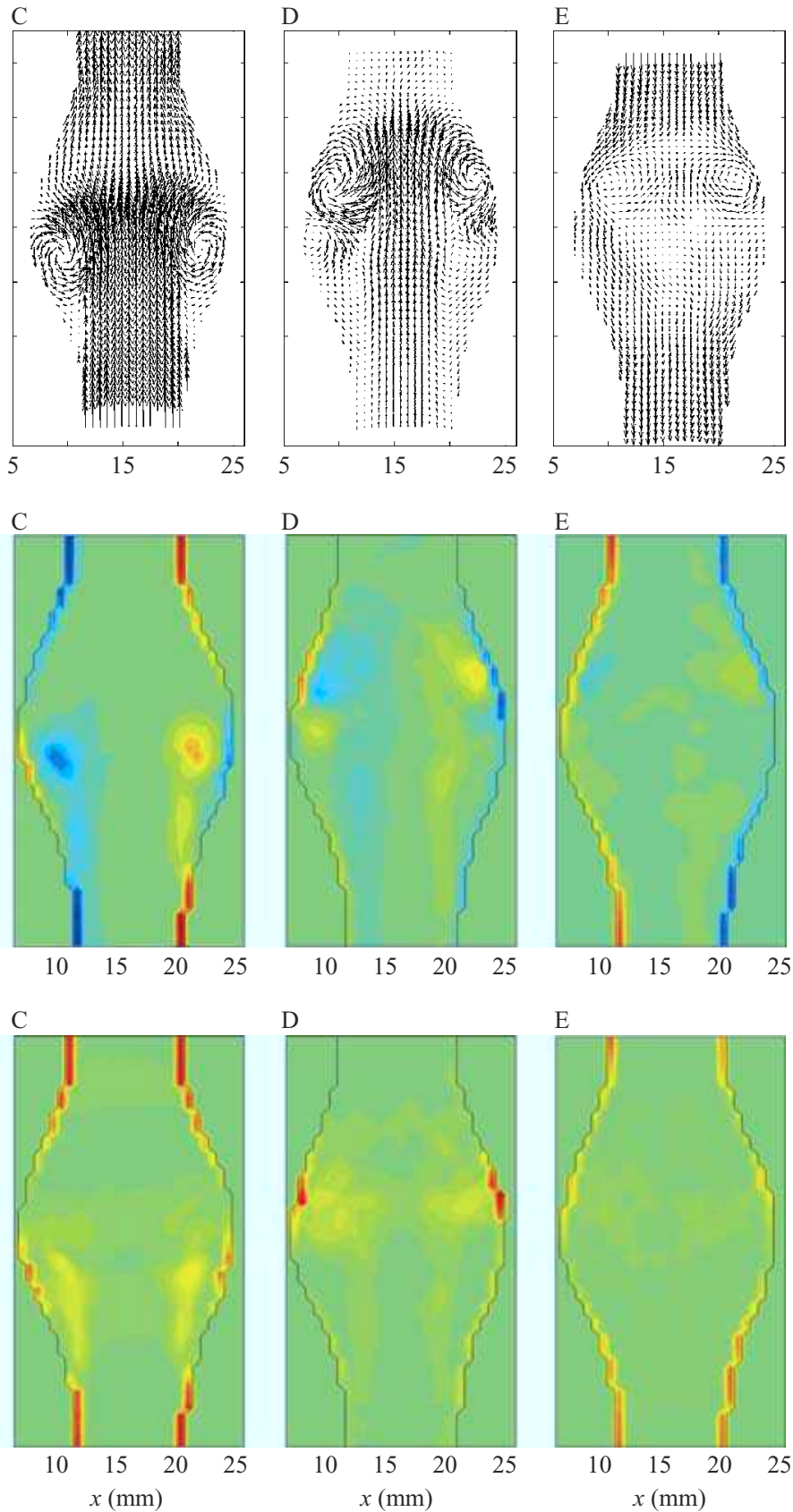

D

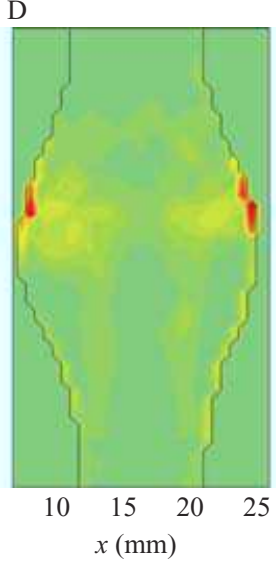

E

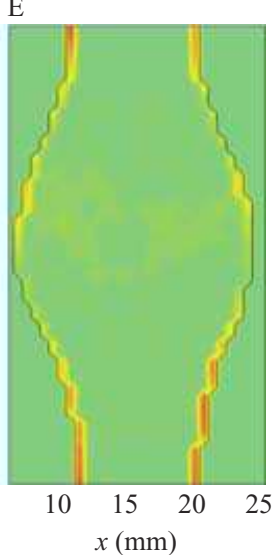

FIGURE 7. Phase-averaged $(a)$ velocity, $(b)$ vorticity and $(c)$ stress fields, at times $\mathrm{B}$ to $\mathrm{E}$, measured in model $4(D / d=1.9, L / d=2.9)$. The vorticity and stress fields are nondimensionalized by the peak values occurring in the healthy vessel $\left(610 \mathrm{~s}^{-1}\right.$ for the vorticity and $2.45 \mathrm{~N} \mathrm{~m}^{-2}$ for the stress).

decreasing intensity (figures 5 and $6 \mathrm{E}-\mathrm{F}$ ). In order to quantify the non-axisymmetry, we measured the velocity field in transverse planes of the AAA. Figure 9 shows both the instantaneous and phase-averaged velocity fields measured in the transverse cut located at the point of maximum diameter. At time $\mathrm{C}$, the core of the vortex 
(a)

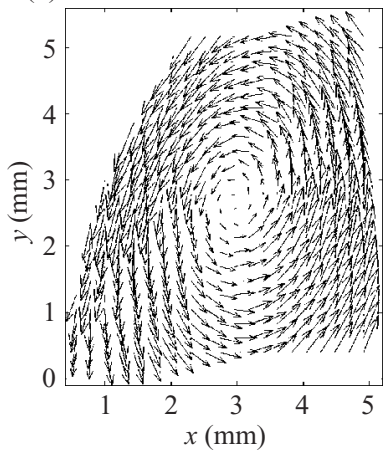

(b)

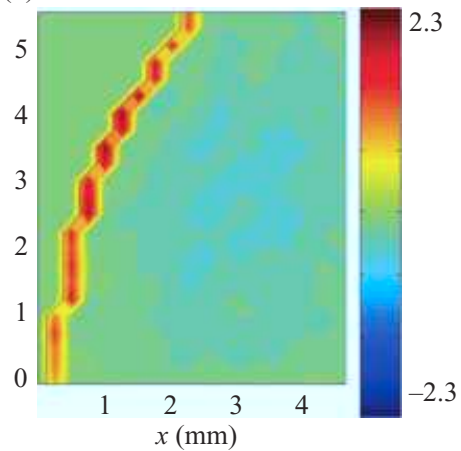

(c)

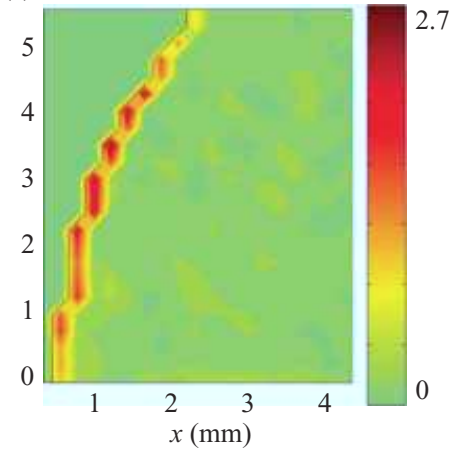

FIGURE 8 . Zoom measurements of the $(a)$ velocity, $(b)$ vorticity and $(c)$ stress fields in the distal area of model $4(D / d=1.9, L / d=2.9)$.
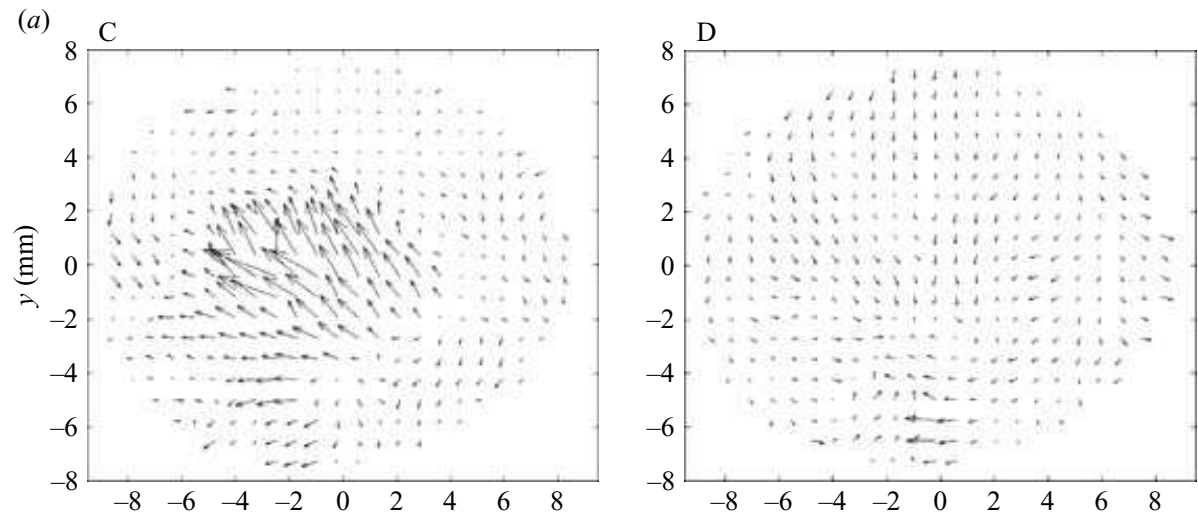

(b)
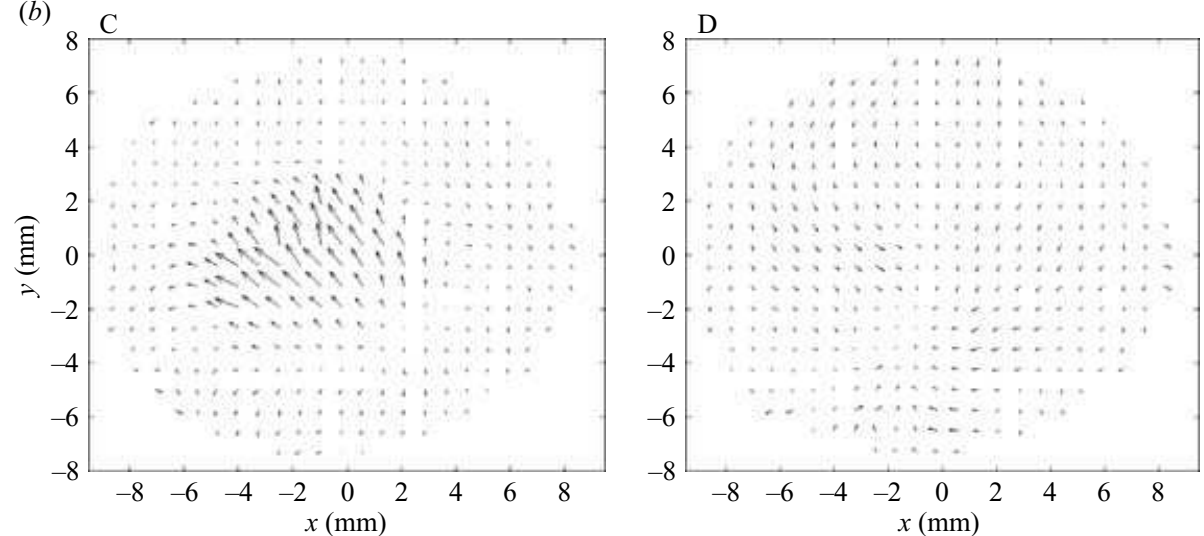

FIGURE 9. (a) Instantaneous and $(b)$ phase-averaged velocity field measured in the transverse cut located at the point of maximum diameter at times $\mathrm{C}$ and $\mathrm{D}$.

ring is located right upstream of the measurement plane. The vortex ring must be slightly inclined, since high outward velocity vectors are measured at the centre of the aneurysm. However, at time D, a negative radial velocity field is induced at the rear of the vortex ring. Figure 9 shows that the loss of symmetry has already occurred at time C. 

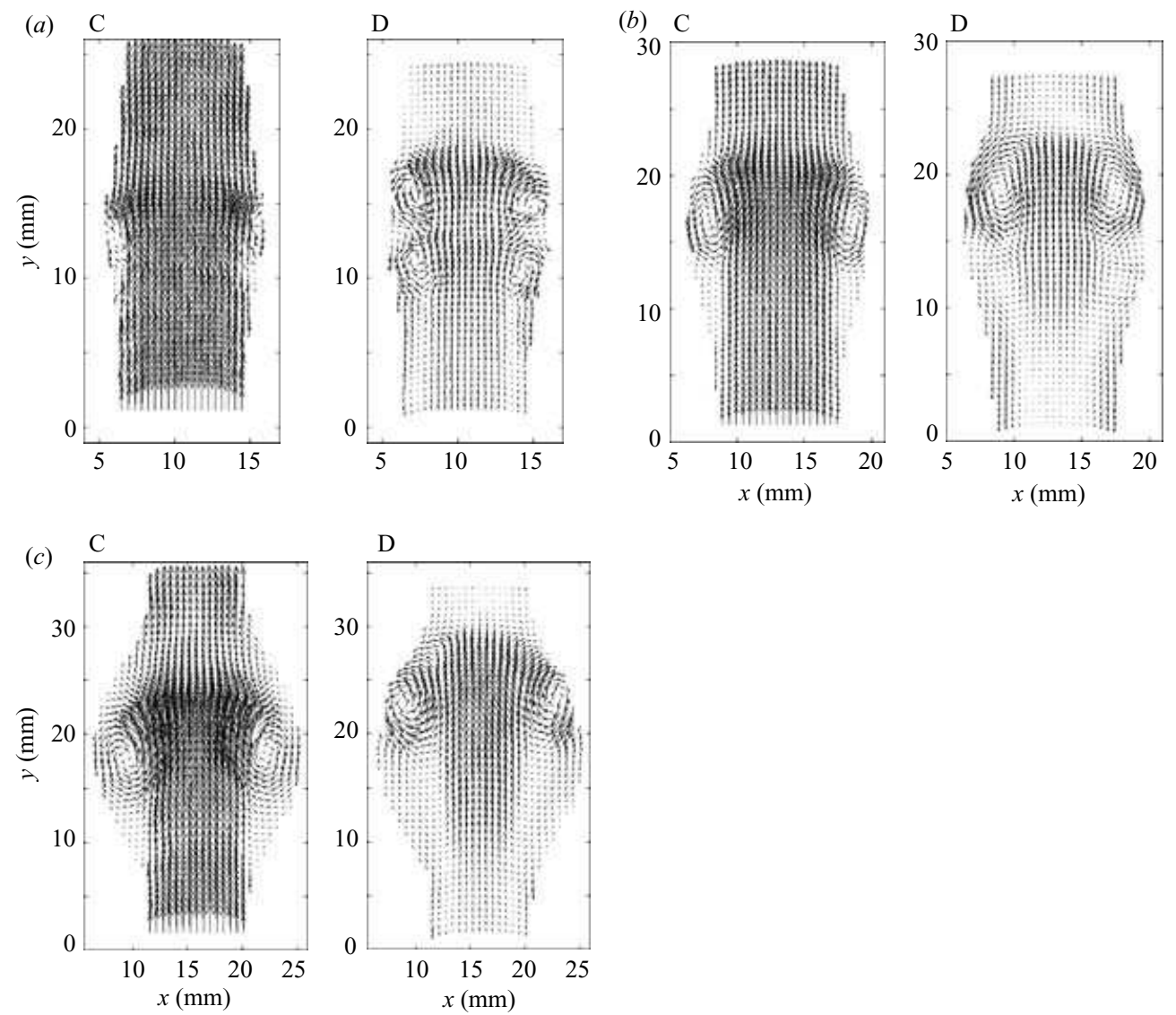

FIgURE 10. Comparison of the phase-averaged velocity field measured with the PIV system in models $1(a), 2(b)$ and $5(c)$ at times $\mathrm{C}$ and $\mathrm{D}$. A small phase-lag between the different experiments is possible, since the frequency of acquisition of the measurements is a harmonic of the frequency of the flow, but the relative phase varies from one experiment to the next.

Near the end of the cardiac cycle corresponding to the resting period of the heart (figure $5 \mathrm{G}-\mathrm{J}$ ), the flow disturbances weaken owing to vortex entanglement (energy cascade) and viscous dissipation and the flow relaxes back to a near stagnant state. At the beginning of the next cycle, some weak perturbations persist from the erratic motion. The initial flow conditions are therefore not perfectly identical for each cycle, and a small cycle-to-cycle variation is observed in our measurements.

\section{Effect of the dilatation parameter}

In order to assess the effect of the dilatation ratio on the flow topology, the flow field in the decelerating portion of the diastole is plotted in figure 10 for three comparable models. Models 1, 2 and 5 have the same aspect ratio as model $4, L / d=2.9$, but their dilatation ratio increases linearly with the model number (see table 1). The corresponding vorticity fields are shown in figure 11 .

It is remarkable that the clinically relevant features of aneurysmal flows, i.e. detachment of the flow and impingement on the distal neck, occur even for a dilatation parameter as low as 1.3 for short aneurysms (figures $10 a$ and 11a). Even an incipient aneurysm is characterized by the formation of recirculating zones close 

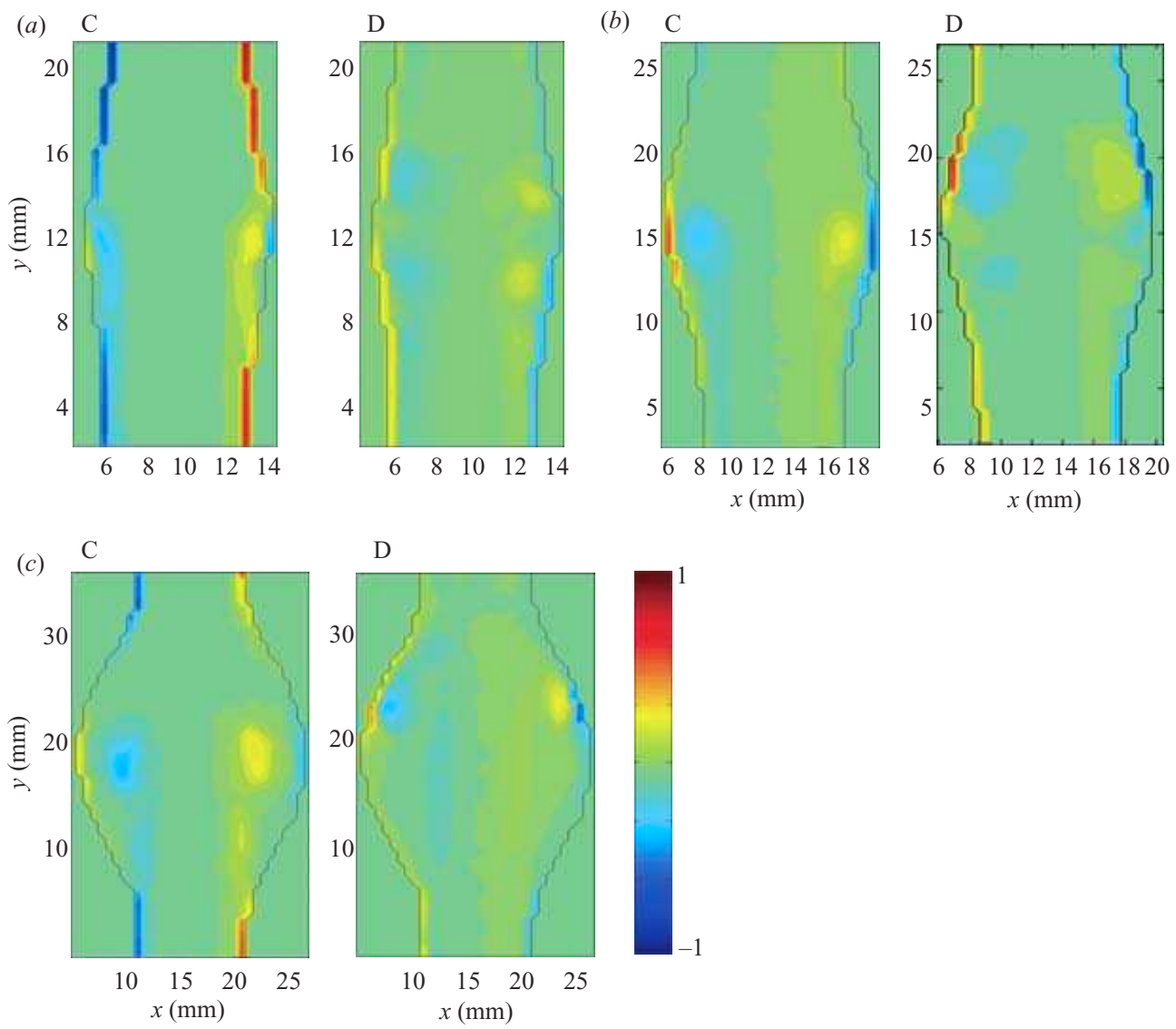

Figure 11. Comparison of the phase-averaged vorticity field measured in models $1(a), 2(b)$ and $5(c)$ at times $\mathrm{C}$ and $\mathrm{D}$, non-dimensionalized by the peak value occurring in the healthy vessel $\left(610 \mathrm{~s}^{-1}\right)$.

to the wall. We can see that the flow separation occurs around the peak systole in all of the models (between times $\mathrm{B}$ and $\mathrm{C}$ in figures 10 and 11). The time at which flow separation occurs, does not seem to depend on the dilatation parameter, contrary to the point of flow separation. As the aneurysm grows in size, the point of flow separation becomes closer to the proximal neck. The size of the detached flow region also increases as the dilatation parameter is increased. When $D / d$ increases, the detachment becomes more massive and a larger vortex ring is generated. A shear instability length scale can be observed in the shear layer for the small dilatation ratio models. It is of the order of the vessel radius $a$ (figure $10 a, b$ ). It is, however, impossible to conclude whether this length scale is the primary one or whether vortex pairing has already occurred.

\section{Effect of the aspect ratio}

Figure 12 features the phase-averaged velocity fields at times C and D for models 11,6 and 1 . The models have decreasing aspect ratios, but they all have the same dilatation ratio $(D / d=1.3)$. 
(a)

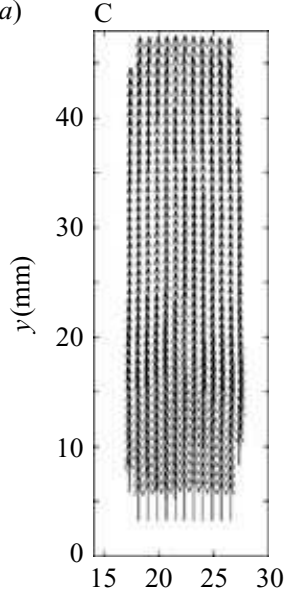

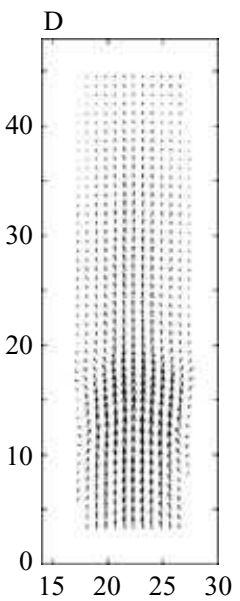
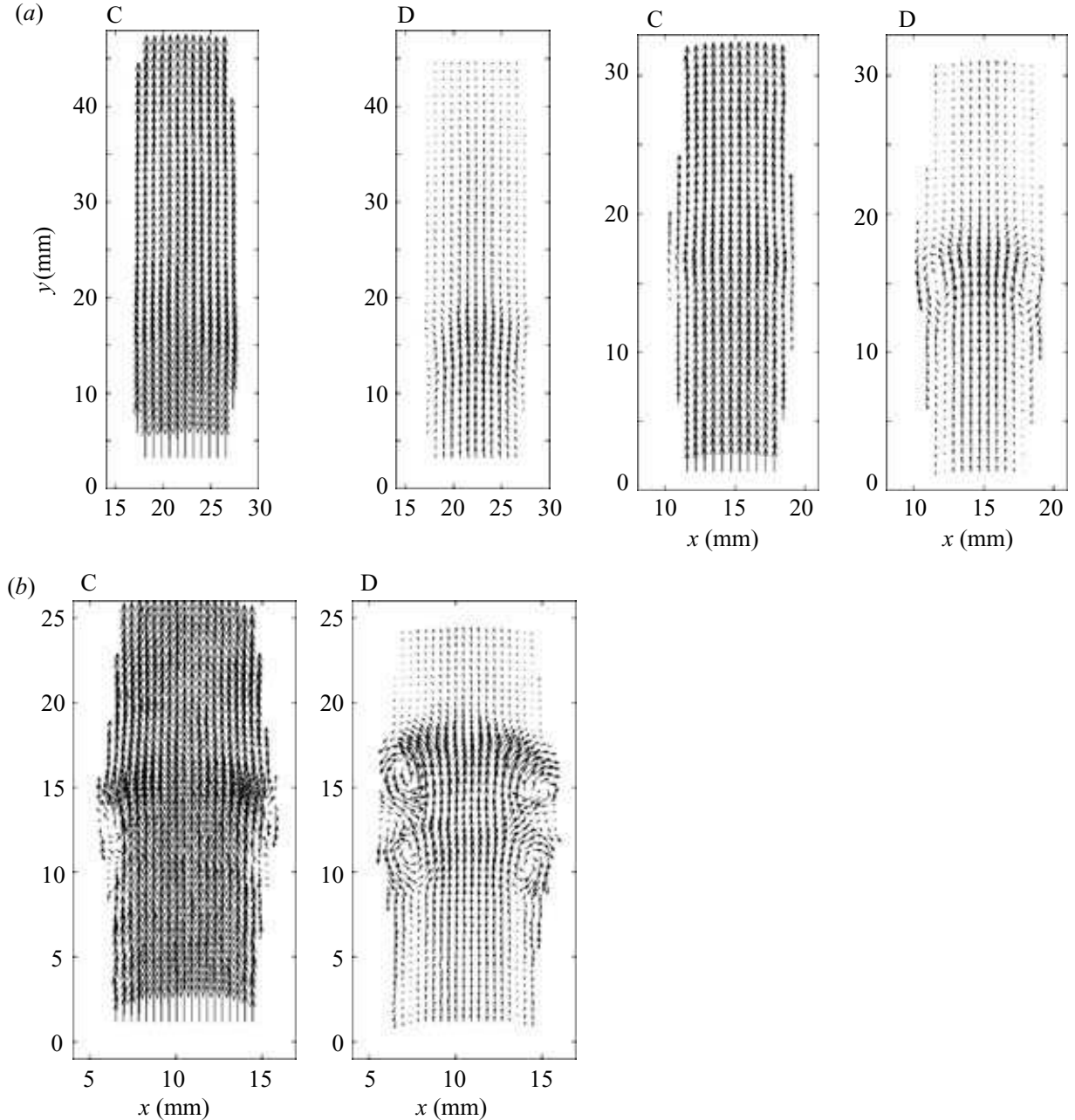

FIGURE 12. Comparison of the phase-averaged velocity field measured in models $11(a)$, $6(b)$ and $1(c)$ at times C and D.

A first striking difference is the length travelled by the vortex ring. This length, $l$, measured as the greatest distance between the core of the vortex ring and the proximal neck, was plotted in figure 13 for the different values of $L / d$ and $D / d$ ratios.

The ratio $l / d$ is to be compared with the Strouhal number calculated with the systolic time $t_{\text {syst }}$ (Gharib, Rambod \& Shariff 1998; Stroud, Berger \& Saloner 2000)

$$
S t_{\text {syst }}=\frac{U_{\text {syst }} t_{\text {syst }}}{d} \text {. }
$$

This Strouhal number is the controlling parameter of the vortex shedding. A characteristic systolic velocity can be chosen as half the peak velocity, which would correspond to an average systolic velocity. The Strouhal number is then found to be equal to

$$
S t_{s y s t}=\frac{1}{2} \frac{4 Q_{p}}{\pi d^{2}} \frac{0.16 T}{d}=3
$$




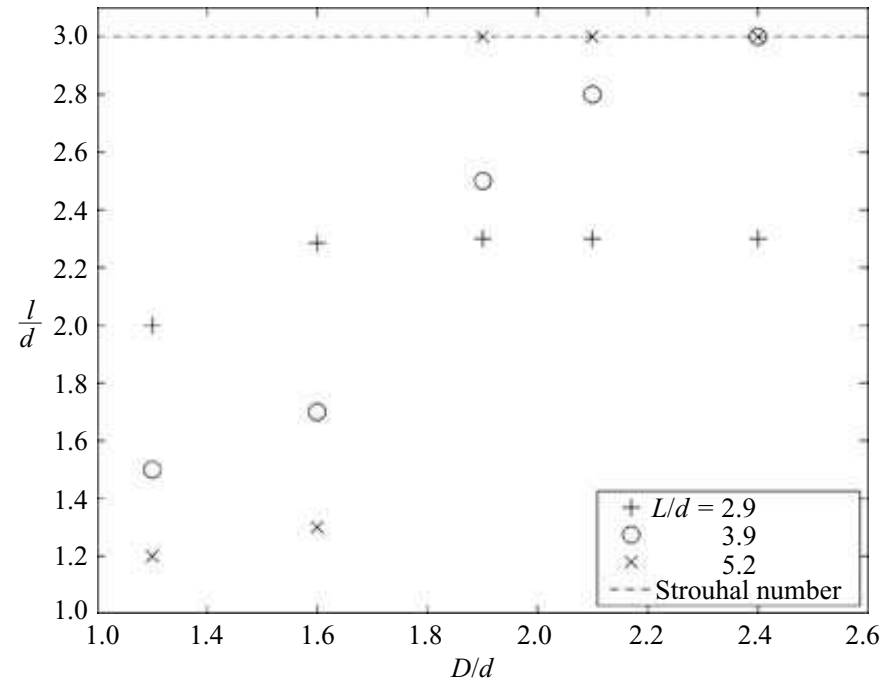

FIGURE 13. Maximum position of the core of the vortex ring inside the AAA plotted as a function of the $D / d$ and $L / d$ ratios.

since the systolic time is about $16 \%$ of the cardiac period $T$. We can see that $l / d$ tends to $S t_{\text {syst }}$, as $D / d$ increases. However, the aspect ratio of the model may limit the evolution of the vortex ring, when the length available to the vortex ring to propagate is less than $3 d$, as seen for $L / d=2.9$. In models 2 to 5 , the cores of the vortex ring do not go beyond $y / d=2.3$, since this location corresponds to the point of impingement on the distal wall. Apart from the case of small aspect ratios, where $L / d$ is a limiting parameter, the length travelled by the vortex ring inversely scales with $L / d$.

The second difference is the time at which flow separation occurs in the cycle. Whereas the flow separates around the peak systole in model 1 (between times B and $\mathrm{C}$ in figure 12c), the separation is delayed to time $\mathrm{C}$ in model 6 (figure $12 b$ ) and even further to time D in model 11 (figure 12a). Since the separation occurs later in the deceleration phase for higher aspect ratios, the generated vortex ring has a reduced strength. This implies that longer aneurysms are less pathological than short ones. An increased length delays the appearance of 'disturbed flow' conditions and drastically reduces the magnitude of the vortex strength. As far as the geometrical parameters are concerned, these results correlate well with those found by Hatakeyama, Shigematsu $\&$ Muto (2001), who characterized the risk factors for rupture as the diastolic pressure, the ratio $D / L$ and the expansion rate of the aneurysm.

4.2. Results of the parametric study of the wall shear stresses in $A A A$ Comparison of the WSS in a typical AAA with the reference case

We discuss here the results of the parametric study, concentrating now on the spatial and temporal changes in wall shear stresses, which occur as the AAA enlarges. We shall first present the results obtained in model 4 to analyse the general changes that occur in a typical established aneurysm as compared to a healthy abdominal aorta. The effects of the dilatation parameter and aspect ratio will then be discussed in the following subsections.

The wall shear stresses are defined as

$$
W S S=2 \mu(\boldsymbol{\sigma} \cdot \boldsymbol{n}) \cdot \boldsymbol{t},
$$



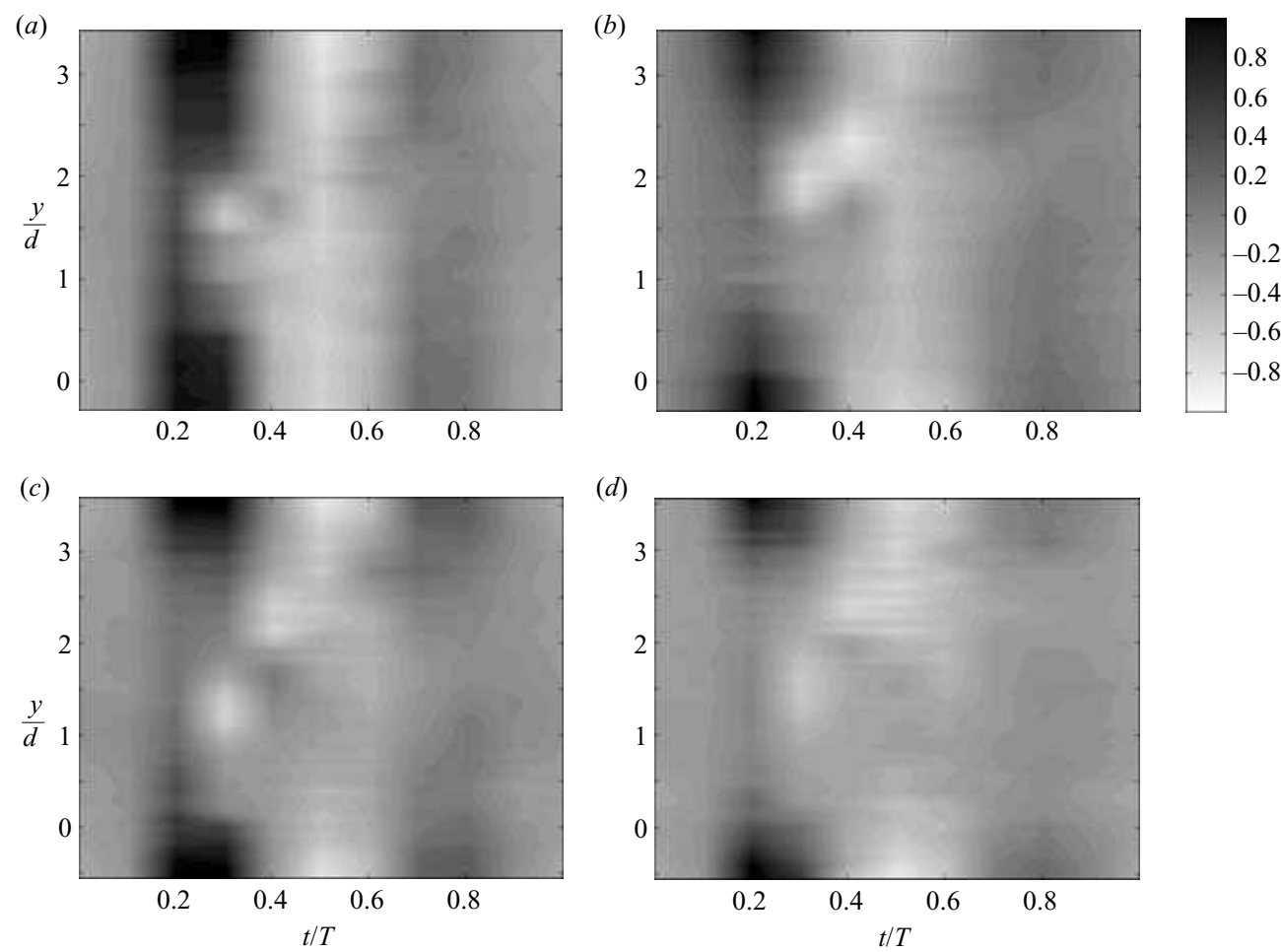

Figure 14. Time evolution of the phase-averaged wall shear stresses in models $1(a), 2(b)$, $4(c)$ and $5(d)$. The wall shear stresses have been non-dimensionalised by the peak value in a healthy abdominal aorta $\left(4.9 \mathrm{~N} / \mathrm{m}^{-2}\right) \cdot y / d=0$ is located at the proximal neck for each of the models. On the time scale, $t / T=0.1$ corresponds to time $\mathrm{A}, t / T=0.2$ to time $\mathrm{B}$, etc.

$\boldsymbol{n}$ and $\boldsymbol{t}$ being, respectively, the normal and tangential unit vectors and

$$
\sigma=\frac{1}{2} \mu\left[\nabla \boldsymbol{u}+\nabla \boldsymbol{u}^{T}\right]
$$

the rate of deformation tensor for a Newtonian fluid. In Cartesian two-dimensional coordinates, the WSS takes the form

$$
W S S=2 \mu\left[\sigma_{x y}\left(n_{x}^{2}-n_{y}^{2}\right)+\left(\sigma_{y y}-\sigma_{x x}\right) n_{x} n_{y}\right] .
$$

The phase-averaged WSS, non-dimensionalized by the peak WSS of a healthy abdominal aorta, is shown in figure $14(c)$ for the case of model 4 . In the systole $(t / T=0.2)$, the WSS decreases as the local diameter increases. The WSS follows a similar trend to the velocity, which inversely scales with the diameter in order to conserve mass. This is because the flow is fully attached to the walls at systole. At $t / T=0.3$, the flow detaches from the proximal neck $(y / d=0)$ creating a region of zero WSS in the range $0 \leqslant y / d \leqslant 0.8$. The region of large negative WSS around $y / d=1.2$ is due to the presence of the core of the large primary vortex ring. At $t / T=0.4$, the vortex has travelled to $y / d=2.1$, but the fact that the measurements are taken at discrete times prevents us from following more closely the vortex ring, during its evolution through the AAA. At $t / T=0.4$, the vortex ring impinges on the distal wall. However, as discussed in the previous subsection, the WSS value is underestimated at 
(a)

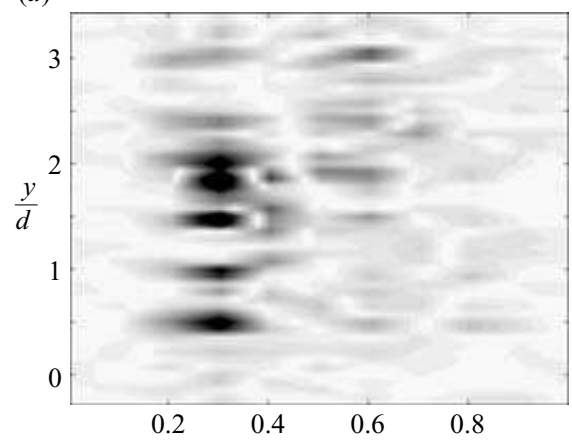

(c)

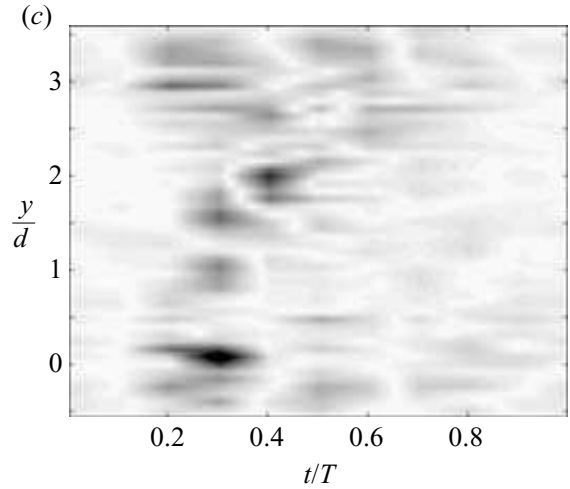

(b)

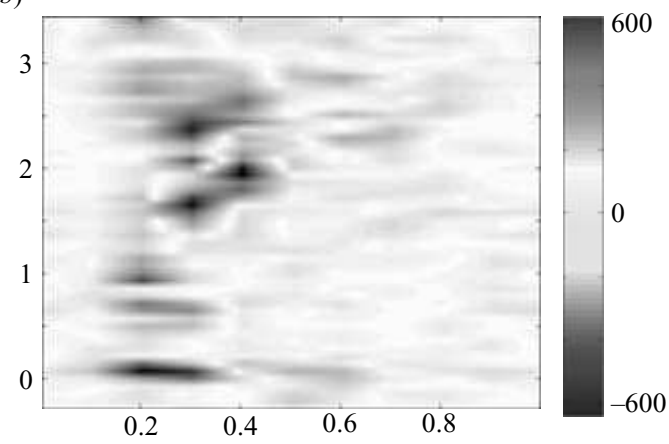

(d)

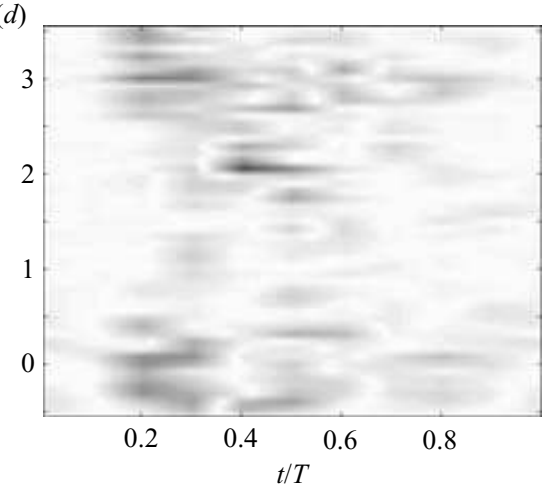

FIGURE 15. Time evolution of the gradient of the phase-averaged wall shear stresses in models $1(a), 2(b), 4(c)$ and $5(d)$, measured in $\mathrm{N} \mathrm{m}^{-3} \cdot y / d=0$ is located at the proximal neck for each of the models. On the time scale, $t / T=0.1$ corresponds to time $\mathrm{A}, t / T=0.2$ to time $\mathrm{B}$, etc.

the point of impact, because of the very small size of the boundary layer and of the resolution of our measurements. Furthermore, the presence of the primary vortex ring so close to the wall induces the roll-up of the boundary layer into a counter-rotating vortex of smaller size, which can be observed at $y / d=1.5, t / T=0.4$. Upstream of it, the wall still experiences very low WSS $(W S S \simeq 0)$. At the end of the diastole as well as in the resting period of the cycle, the walls of the entire aneurysm are dominated by very low WSS.

The gradients of the phase-averaged WSS are plotted in figure 15(c). They have not been non-dimensionalized by the peak value in a healthy abdominal aorta, since the gradients are very small in this case. Strong gradients are observed at the proximal and distal necks at times $t / T=0.2$, when the changes in WSS are maximum at the necks and $t / T=0.3$, when the flow detaches in the aneurysm. The formation of the vortex ring also induces very strong gradients just upstream and downstream of it $(t / T=0.3-0.4)$. The locations of these regions of high gradients therefore move along with the vortex ring, affecting different parts of the wall over time; but the time, when most of the aneurysm wall experiences strong gradients, occurs after the flow massively detaches from the wall $(t / T=0.3)$.

The $W S S_{\text {mean }}$, defined in $\S 3.1$ along with $W S S_{\text {mag }}$ and the $O S I$ index, sharply drops after the proximal neck to almost zero and remains very low in the range 
(a)

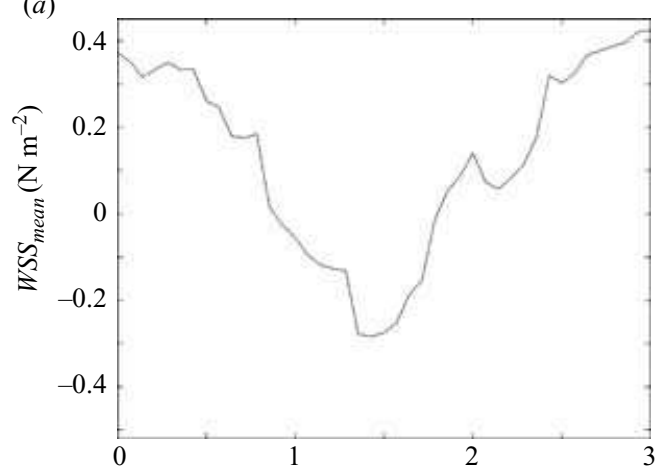

(c)

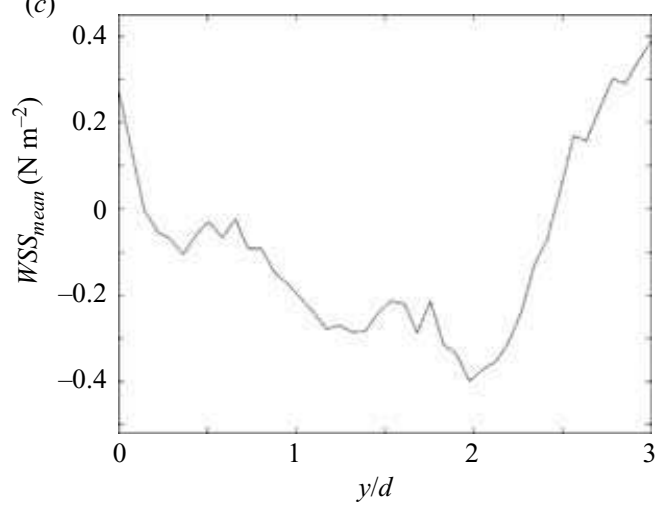

(b)

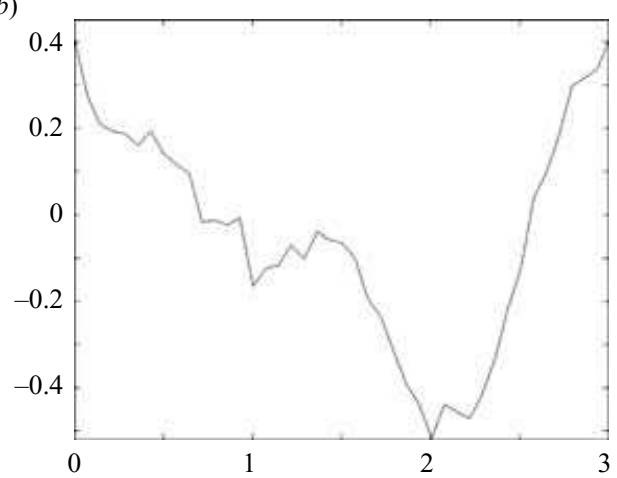

(d)

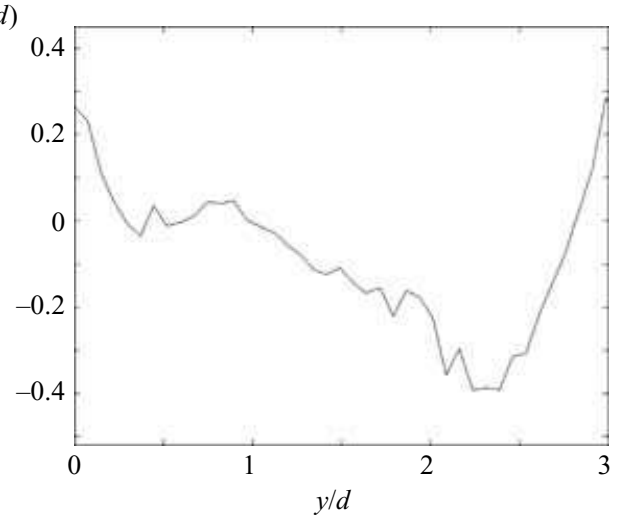

FIGURE 16. Evolution of $W_{\text {SS }}$ mean along the aneurysmal wall in models $1(a), 2(b), 4(c)$ and $5(d)$. The results are shown only inside the AAA.

$0.1 \leqslant y / D \leqslant 0.7$. This can be seen in figure $16(c)$, which shows the $W S S_{\text {mean }}$ for model 4. The $W S S_{\text {mean }}$ then reaches larger negative values, the negative peaks correlating with the presence of the vortex ring, at times $t / T=0.3$ and 0.4 . We can see that, on average, most of the aneurysm wall is subjected to negative $W_{S S} S_{\text {mean }}$ of small amplitude in the proximal half, but large amplitude in the distal half. The magnitude of the WSS (WSS mag $)$ is less sensitive to the presence of the vortex ring. It decreases in the proximal half and increases back in the distal half.

The $O S I$ index spots out the regions of oscillatory flows $(O S I \sim 0.5)$ as well as the regions of forward $(O S I \sim 0)$ or reversed $(O S I \sim 1)$ flow. The region of very low $W S S_{\text {mean }}$ observed in model $4(0.1 \leqslant y / d \leqslant 0.7)$ is characterized by an OSI index close to 0.5 (figure $17 c$ ). In the regions of high negative $W S S_{\text {mean }}$, the $O S I$ index further increases to reach 0.8 over a large portion of the AAA $(1.3 \leqslant y / d \leqslant 2.2)$. Most of the aneurysm wall experiences an $O S I$ index larger than 0.5 , which shows that reversed flow conditions are dominant.

Effect of the dilatation parameter on the WSS

In the systole, the decrease of the WSS becomes more pronounced with increasing $D / d$ ratio (figure 14). As $D / d$ increases, the point of flow separation becomes closer to the proximal neck $(y / d=0.8$ for model 1 to be compared to $y / d=0.1$ for models 2 , 4 and 5). So the $W S S_{\text {mean }}$ drops more abruptly to near zero values as $D / d$ increases 

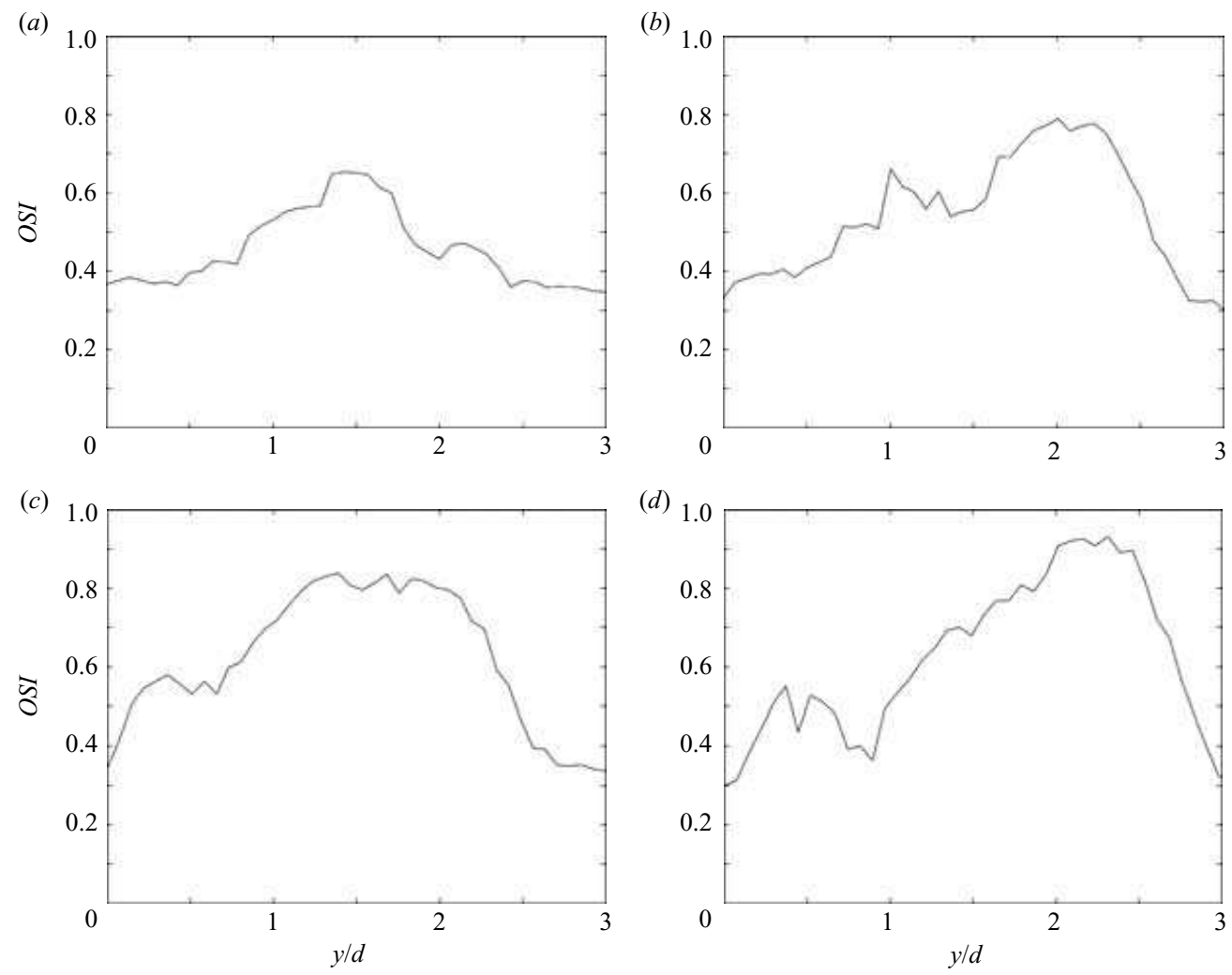

FigURE 17. Evolution of the OSI index along the aneurysmal wall in models $1(a), 2(b), 4(c)$ and $5(d)$. The results are shown only inside the AAA.

(figure 16). Finally, the size of the recirculation region is seen to increase with $D / d$, increasing from a length equivalent to $1 d$ in model 1 , to $2 d$ in model 2 , to the whole length of the aneurysm $(2.8 d)$ in model 5 . The increase in the size of the detached region leads to an increase in the section of the vessel wall subjected to a reversed flow, characterized by a negative mean WSS (figure 16) and an OSI index greater than 0.5 (figure 17). The accentuation of the drop in WSS in the systole and the increase in size of the regions subjected to very low WSS are responsible for the gradual decrease in $W S S_{m a g}$ as $D / d$ increases. The average value of the $W S S_{\text {mag }}$ inside the AAA drops from $1.1 \mathrm{~Pa}$ in model 1 , to $0.75 \mathrm{~Pa}$ in model 2, to $0.60 \mathrm{~Pa}$ in model 4 and finally to $0.37 \mathrm{~Pa}$ in model 5 . The decrease in $W S S_{m a g}$ causes an increase in the maximum of the OSI index (figure 17), so that the average value of the index dramatically increases as $D / d$ increases.

The time at which the flow separates from the wall does not depend strongly on the dilatation parameter. Detachment occurs for all the models at the initiation of the deceleration portion of the cycle, between $t / T=0.2$ and 0.3 , which corresponds to times $\mathrm{B}$ and $\mathrm{C}$ in figure $5 \mathrm{~K}$.

\section{Effect of the aspect ratio on the WSS}

For a same dilatation parameter $D / d$, the main effect of increasing the aspect ratio of the aneurysm is to make all the above described changes in the patterns of WSS less pronounced. Figure 18(a) shows the spatial and temporal evolution of the WSS 
(a) (i)

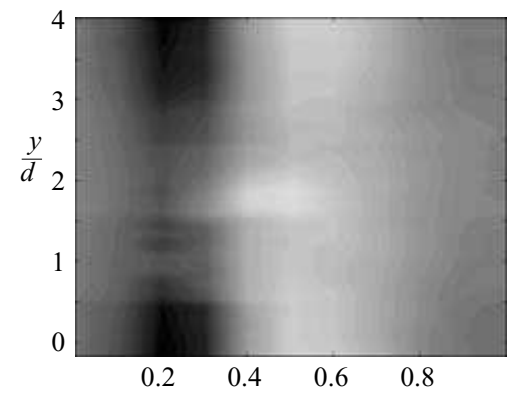

(b) (i)

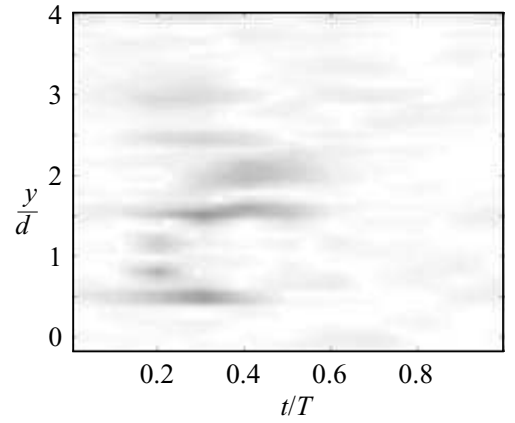

(c) (i)

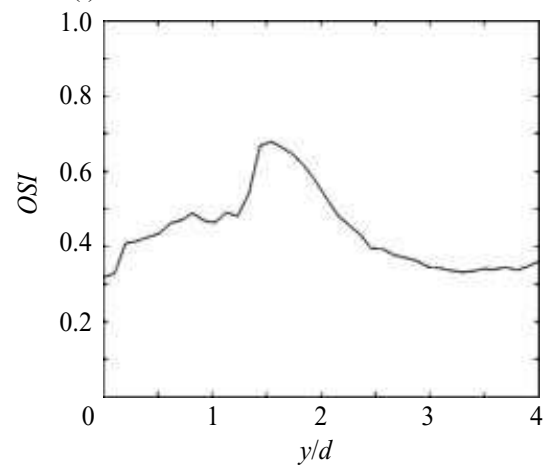

(ii)

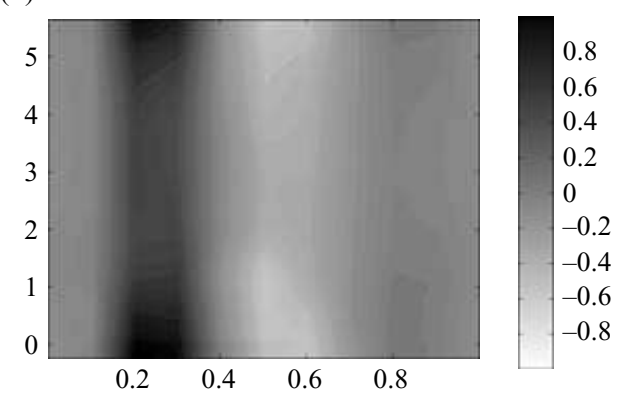

(ii)

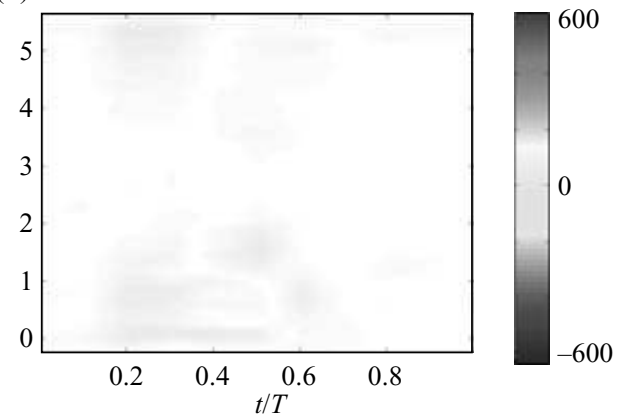

(ii)

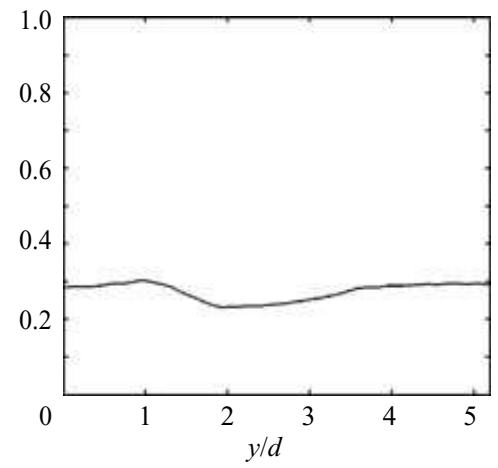

FIGURE 18. (a) Time evolution of the phase-averaged wall shear stresses, non-dimensionalized by the peak WSS in a healthy abdominal aorta $\left(4.9 \mathrm{~N} \mathrm{~m}^{-2}\right)$ in models $6-L / d=3.9$ and $11-$ $L / d=5.2$. (b) Time evolution of the gradient of the phase-averaged stresses in models 6 and 11. (c) Evolution of the OSI index along the aneurysmal wall in models 6 and 11. The results are shown only inside the aneurysmal cavity.

in models 6 and 11 , which have the same dilatation ratio $(D / d=1.3)$ as model 1 , but an increasing aspect ratio $(L / d=3.9$ and 5.2). The results are therefore to be compared with figure 14(a). In the systole, the effect of the dilatation is visible for all the models. However, we can see that the flow separation is so incipient in model 11 that no appreciable vortex is seen forming.

As $L / d$ increases, the flow separation is delayed (figure 18a) and occurs only at $t / T=0.3$ for model 6 and at $t / T=0.4$ for model 11 . The vortex ring, generated at a later time in the cardiac cycle, is therefore weaker in intensity, inducing smaller 
negative WSS. The delay of the flow separation to instances when the WSS are smaller and the decrease in intensity of the vortex ring explain the very large decrease in WSS gradients measured as $L / d$ increases (figure $18 b$ ). Note that, in model 11 , the GWSS drop to negligible values, the flow conditions approaching those in a healthy aorta. Similarly, the $O S I$ index remains in the healthy range (figure $18 c($ ii)). For the rest of the discussion, we will now set model 11 aside, since it is the only model not to exhibit the classical characteristics of the flow in an AAA.

We can see that the relevant parameters, such as the $W S S_{\text {mean }}$ or the OSI index, have a similar variation in space for models 1 and 6 (figure $17 a$ and figure $18 c(\mathrm{i})$ ). The values are practically identical in each case and the maximum in OSI occurs at the same location, although the length of the models is different. This shows that the $L / d$ parameter has little effect on the physical processes that occur inside the AAA.

\subsection{Analytical solution for a slowly expanding abdominal aorta}

\section{Haemodynamics}

The study of the changes in the WSS resulting from very small dilatations of the abdominal aorta, prior to their development into an aneurysm, is crucial for a better understanding of the aetiology of the disease. An analytical solution of the flow in an incipient aneurysm, such as the one studied in model 11, would therefore be a useful tool to study the evolution of AAAs in a systematic way.

We can obtain an analytical solution for the WSS by extending the case of a straight pipe, shown in $\S 3$ to a slowly expanding pipe. In this case, the radial velocity, $u_{r}(y, r, t)$, cannot be neglected. Let $\mathscr{L}$ be the characteristic length along which the local radius $a(y)$ varies, i.e. $\mathscr{L}=a_{0} \mathrm{~d} a / \mathrm{d} y$. In the case of an incipient aneurysm, we can assume that the changes of the $y$-velocity, $v^{*}\left(y^{*}, r^{*}, t^{*}\right)$, are slow along $y$. A new variable $Y^{*}=\epsilon y^{*}$ is therefore introduced, where the small parameter $\epsilon$ is defined as $\epsilon=\mathscr{L} / a_{0}$. We also define a new radial velocity, $U_{r}^{*}$, such that $u_{r}^{*}=\epsilon U_{r}^{*}$ with $U_{r}^{*}$ of order 1 and a new pressure $P^{*}=\epsilon p^{*}$. At the first order, the momentum equations are decoupled and reduce to those corresponding to the straight pipe. The dimensionless longitudinal velocity $v^{*}\left(Y^{*}, r^{*}, t^{*}\right)$ is given by

$$
v^{*}=\frac{\operatorname{Re} G_{0}^{*}\left(Y^{*}\right)}{4}\left(R^{* 2}-r^{* 2}\right)+\sum_{n=1}^{\infty} \frac{\operatorname{Re} G_{n}^{*}\left(Y^{*}\right) R^{* 2}}{\mathrm{i} \alpha_{n}^{2}}\left(1-\frac{J_{0}\left(\mathrm{i}^{3 / 2} \alpha_{n} r^{*} / R^{*}\right)}{J_{0}\left(\mathrm{i}^{3 / 2} \alpha_{n}\right)}\right) \mathrm{e}^{\mathrm{i} n t^{*}},
$$

where $R^{*}\left(Y^{*}\right)=a\left(Y^{*}\right) / a_{0}$ is the dimensionless local radius, $\alpha_{n}\left(Y^{*}\right)=a\left(Y^{*}\right) \sqrt{n \omega / v}$ the local Womersley number corresponding to the $n$th harmonic of the pulsation $\omega$ and $G_{n}^{*}\left(Y^{*}\right)$ the $n$th Fourier coefficient of the pressure gradient $\partial P^{*} / \partial Y^{*}$.

The pressure coefficients $G_{n}^{*}\left(Y^{*}\right)$ are then calculated taking into account that the flow-rate is conserved for each Fourier mode at any location $Y^{*}$. The flow-rate components $Q_{n}^{*}$ are independent of $Y^{*}$, thus

$$
G_{0}^{*}\left(Y^{*}\right)=\frac{8}{\pi \operatorname{Re} R^{* 4}} Q_{0}^{*} ; \quad G_{n}^{*}\left(Y^{*}\right)=\frac{\mathrm{i} \alpha_{n}^{2}}{\pi \operatorname{ReR}^{* 4}\left[1-F\left(\alpha_{n}\right)\right]} Q_{n}^{*} .
$$

The radial velocity takes the form

$$
\begin{aligned}
U_{r}^{*}=\frac{2 Q_{0}}{\pi R^{* 2}} & \frac{\mathrm{d} R^{*}}{\mathrm{~d} Y^{*}} \frac{r^{*}}{R^{*}}\left(1-\frac{r^{* 2}}{R^{* 2}}\right) \\
& +\sum_{n=1}^{\infty} \frac{Q_{n}}{4 \pi R^{* 2}} \frac{\mathrm{d} R^{*}}{d Y^{*}} \frac{\mathrm{i} \alpha_{n}^{2} F\left(\alpha_{n}\right)^{2}}{\left(1-F\left(\alpha_{n}\right)\right)^{2}}\left(\frac{r^{*}}{R^{*}}-\frac{J_{1}\left(\mathrm{i}^{3 / 2} \alpha_{n} r^{*} / R^{*}\right)}{J_{1}\left(\mathrm{i}^{3 / 2} \alpha_{n}\right)}\right) \mathrm{e}^{\mathrm{i} n t^{*}} .
\end{aligned}
$$




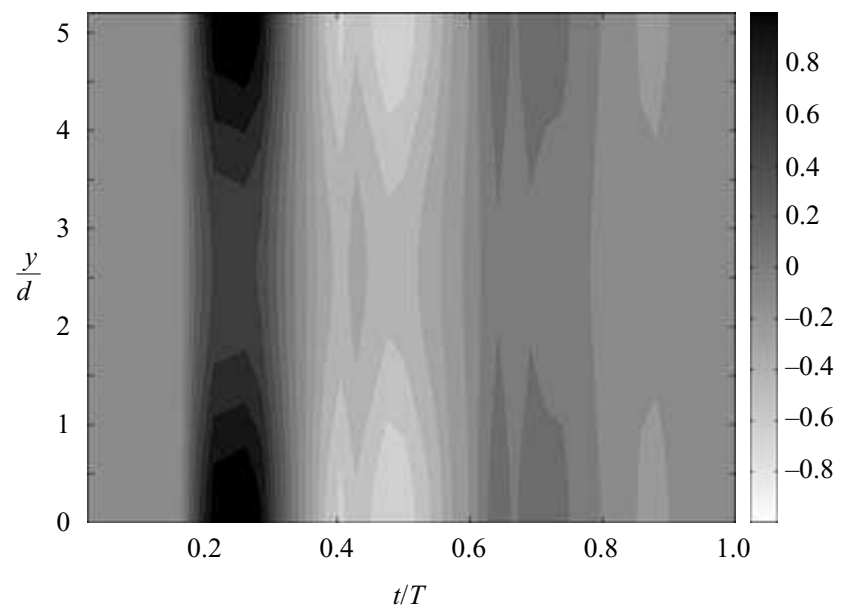

FIGURE 19. Wall shear stresses calculated with the slowly expanding aneurysm model $(D / d=1.3, L / d=5.2)$. The WSS have been non-dimensionalized by the peak value in the healthy vessel $\left(4.9 \mathrm{~N} \mathrm{~m}^{-2}\right)$. The WSS distribution is very similar to that measured in model 11 (figure $18 a($ ii) $)$.

\section{Wall shear stresses}

At the first order, the wall shear stresses are given by equation (3.8), the wall being located at $r^{*}=R^{*}$. The WSS can be calculated for the flow rate given by the pump in the case of model 11 . Similarly to the procedure in $\S 3$, we can calculate the Fourier coefficients of the pressure gradients, $G_{n}^{*}\left(Y^{*}\right)$, from the Fourier decomposition of the flow rate. The WSS profile given by the numerical model (figure 19) can be compared to figure 18(a)(ii), which shows the experimental result in model 11. The two plots are very similar. The difference comes again from the smoothing effect that was previously shown in the experiment.

\section{Discussion}

It has been long known that the structural function of the intimal layer of the abdominal aorta (comprising the endothelial cells) is sensitive to the local haemodynamic parameters. Experiments, in which the temporal distribution of shear stresses applied to the endothelial cells could be carefully controlled, have shown that the endothelial behaviour depends not only on the magnitude of the shear stresses, but also on their spatial and temporal variations. These mechano-transduction mechanisms have been postulated to play a key role in vasoregulation and in the localization of intima hyperplasia and intima lesions in regions of disturbed flow conditions, i.e. regions where the WSS and GWSS differ from the healthy conditions. Thus, the characterization of the changes in the WSS resulting from the enlargement of the artery is essential to understand the aetiology of AAAs as well as the role that the haemodynamics plays in its progression.

Our comparative measurements of the spatial and temporal distribution of the wall shear stresses in idealized models of AAA have shown that the flow inside the aneurysm is characterized by the formation of regions of larger and lower amplitude of wall shear stresses than in the healthy vessel, as well as regions of large gradients of wall shear stresses, which are virtually non existent in the healthy case. We have found 


\begin{tabular}{lccrrrr} 
& $A_{-1}$ & \multicolumn{1}{c}{$A_{0}$} & \multicolumn{1}{c}{$A_{1}$} & \multicolumn{1}{c}{$A_{2}$} & \multicolumn{1}{c}{$A_{3}$} & \multicolumn{1}{c}{$A_{4}$} \\
& $y / d=-0.4$ & 0 & 0.75 & 1.5 & \multicolumn{1}{c}{2.25} & \multicolumn{1}{c}{3} \\
$W S S_{\text {max }} \mathrm{N} \mathrm{m}^{-2}$ & 4.50 & 2.80 & 1.20 & 0.80 & 1.00 & 3.30 \\
$W S S_{\text {min }} \mathrm{N} \mathrm{m}^{-2}$ & 2.50 & -2.50 & -1.50 & -1.00 & -2.00 & -2.00 \\
$W S S_{\text {mean }} \mathrm{N} \mathrm{m}^{-2}$ & 0.44 & 0.28 & -0.09 & -0.23 & -0.24 & 0.39 \\
$W S S_{\text {mag }} \mathrm{N} \mathrm{m}^{-2}$ & 1.46 & 0.88 & 0.46 & 0.38 & 0.60 & 1.20 \\
OSI & 0.35 & 0.34 & 0.60 & 0.81 & 0.70 & 0.34
\end{tabular}

TABLE 2. Comparison of different characteristic quantities, $W S S_{\max }, W S S_{\text {min }}, W S S_{\text {mean }}$, $W S S_{m a g}$ and $O S I$, at six positions along the AAA in model 4: $A_{-1}(y / d=-0.4), A_{0}(y / d=0)$, $A_{1}(y / d=0.75), A_{2}(y / d=1.5), A_{3}(y / d=2.25)$ and $A_{4}(y / d=3)$.

that, even at large values of the dilatation parameter, the flow remained attached to the walls during systole as a consequence of the large systolic acceleration, typical of the flow waveform in the aorta. However, we found that, in all cases studied, the flow detaches from the wall during the deceleration period immediately following the peak systole. A large start-up vortex forms and, as it propagates through the aneurysm, secondary vortex rings develop in the associated internal shear layers. With the exception of a small zone affected by the vortex, the vessel walls are then exposed to very low and oscillating wall shear stresses. The flow detachment and the impingement of the vortex ring on the distal end of the AAA cause the formation of regions along the wall with large spatial gradients of wall shear stresses. In addition, we showed that a region of sustained gradients of wall shear stresses always forms for aspect ratios smaller than the systolic Strouhal number, $S t_{s y s}$, when the primary vortex ring impinges on the distal end. During the diastole, the flow reversal causes a transition to a state of weak turbulence, which is subsequently dissipated over the resting period of the cardiac cycle.

These changes in the flow characteristics result in very large changes in the spatial and temporal distribution of the WSS acting on the endothelial cells as compared to those acting on the healthy aorta. Depending on their location along the aneurysm wall, the EC experience very different patterns of WSS and GWSS as the AAA enlarges. Our measurements show the existence of two main regions, the location and size of which change as the aneurysm grows: the detached region and the reattachment region. The first region is located approximately in the proximal half of the AAA and is dominated by a large decrease in the magnitude of the WSS, as shown in table 2 . The WSS profile evolves from the highly pulsatile waveform measured in the healthy vessel $(y / d=-0.4)$ to an oscillatory waveform of small amplitude $\left( \pm 1 \mathrm{~N} \mathrm{~m}^{-2}\right)$ and zero mean value near the mid-point region $(y / d=0.75)$ to a waveform of even smaller amplitude and negative mean at the point of maximum diameter (figure 20). A large peak in the GWSS occurs at the time of flow separation. However, this peak, owing to its short duration, is unlikely to have an important impact on the function of the endothelial cells.

In contrast, in the reattachment region, comprising the second half of the AAA, we have measured sustained GWSS along with high negative WSS (around $y / d=2.25$ in figure 20). Our measurements show that this second region is subjected to GWSS over $70 \%$ of the duration of the cardiac cycle. Even the distal neck experiences strong fluctuating gradients. Furthermore, the impinging vortex ring produces high wall shear stresses in the reversed flow direction (negative values) at a late time in the cardiac cycle. 

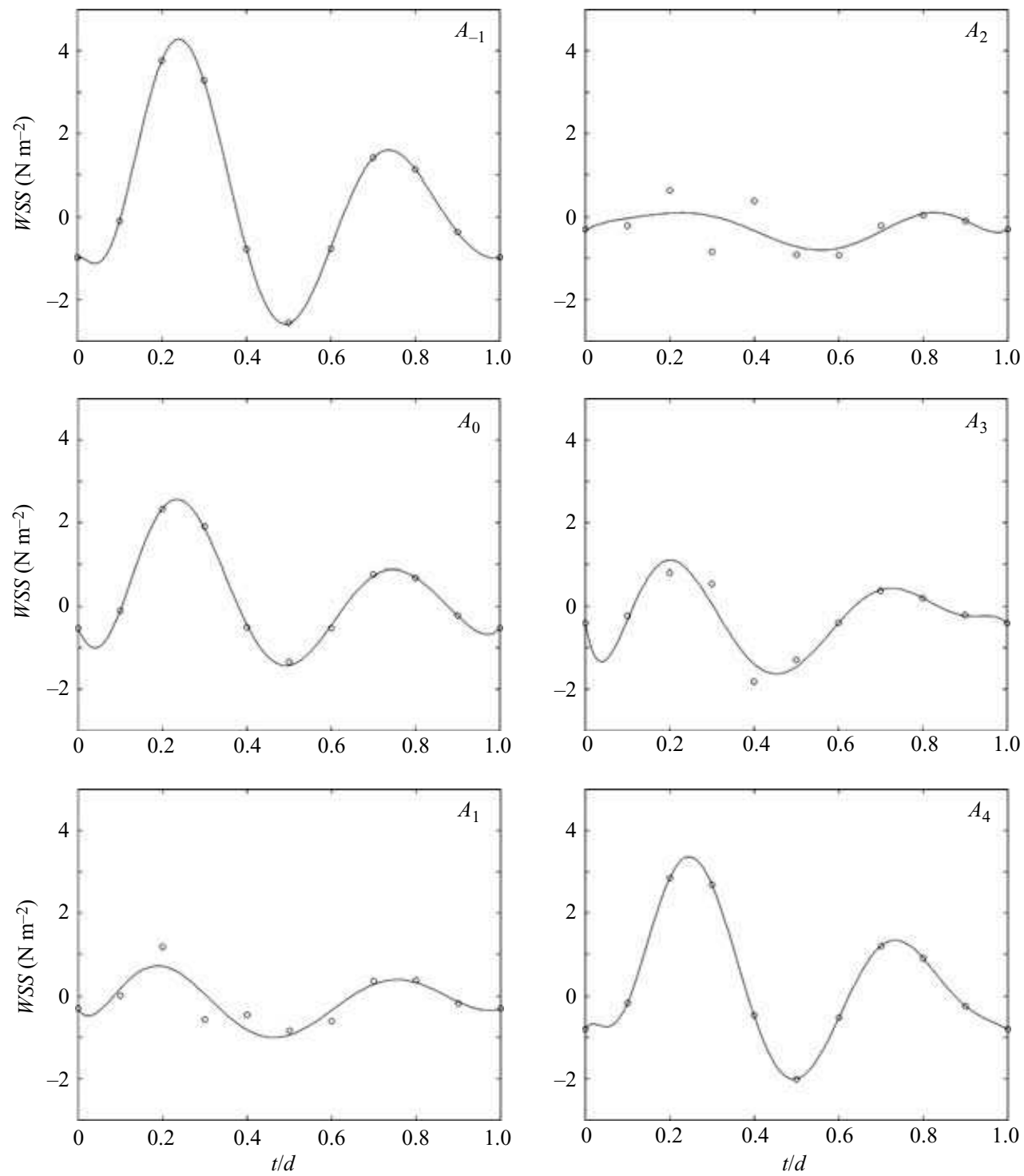

Figure 20. Time evolution of the wall shear stresses at a few locations inside model 4: $A_{-1}$ $(y / d=-0.4), A_{0}(y / d=0), A_{1}(y / d=0.75), A_{2}(y / d=1.5), A_{3}(y / d=2.25)$ and $A_{4}(y / d=3)$.

Our measurements of the spatial and temporal distribution of WSS could provide the basis for the design of endothelial cell experiments, where we could study their proliferation, altered gene expression, cell adhesion as well as the activation of the various biological processes under the realistic flow conditions encountered inside the AAA. These studies will greatly enhance our understanding of the role that the mechanical stimuli play in the aetiology and progression of AAAs.

The flow changes reported here for symmetric AAA are expected to be even more pronounced in in vivo AAAs, since most aneurysms grow non-symmetrically, because of the presence and support of the spinal column. In Salsac et al. (2006), we will show that the basic mechanisms linked to the separation of the flow at the peak systole 
and the roll-up of the vortices are similar for non-axisymmetric AAAs. Since the vortex stretching is stronger and occurs sooner in the non-symmetric case, the flow is likely to be more disturbed. In vivo, the presence of the renal arteries and the lumbar curvature may also have an important effect on the flow and constitute a limitation of our study. The low distal impedance in the renal arteries leads to a suction effect that causes blood at the posterior wall to reverse and to flow back upstream into the renal arteries. Vortices are therefore formed at the entrance of the infrarenal aorta (Moore et al. 1992), modifying partly the flow in the parent vessel to the AAA, especially along the posterior wall. The lumbar curvature may also cause the formation of detached vortices leading to a mechanism similar to that described here for a straight aneurysm. We can infer, in both cases, that the flow field may be phenomenologically similar as far as flow separation, vortex roll-up, and vortex impingement on the wall to the one we conducted. Therefore, although this study was conducted in simplified models of AAA, it captures all the critical flow changes that occur once an aneurysm forms, and provides a much needed tool to analyse quantitatively the effects of the modifications in the mechanical stimuli on the formation and growth of AAAs.

\section{Conclusion}

We have measured systematically the effects of the growth of AAAs on the spatial and temporal distribution of the wall shear stresses. Measurements conducted in rigid symmetric AAA models have shown that flow separation occurs even at very early stages during the AAA formation $(D / d \geqslant 1.3)$. The flow separation and the associated formation of a strong vortex ring and of internal shear layers lead to regions of perturbed stress distribution, which do not exist in a healthy abdominal aorta. For all the aneurysm models characterized by a dilatation ratio greater than 1.5 , the mean WSS consistently drops from the healthy aorta value of $0.40 \mathrm{~N} \mathrm{~m}^{-2}$ to values very close to zero, when averaged over the whole aneurysm length. However, the decrease in the average magnitude of the WSS becomes larger as the dilatation ratio increases.

In terms of WSS patterns, two main regions were identified, the detached region and the reattachment region, the size and location of which change as the aneurysm grows. On the one hand, the detached region, located in the proximal half of the aneurysm,

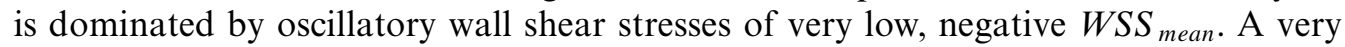
large peak in the GWSS occurs at the location and time of flow separation and reaches about $-600 \mathrm{~N} \mathrm{~m}^{-3}$; but the gradients remain otherwise quite small in the proximal half.

On the other hand, the region of flow reattachment is characterized by large, sustained GWSS and large negative WSS. Most of the distal wall is subjected to GWSS throughout the cardiac cycle. The gradients fluctuate in the region $\pm 400 \mathrm{~N} \mathrm{~m}^{-3}$, the peak values occurring upstream and downstream of the travelling vortex ring. Simultaneously, the $W S S_{\text {mean }}$, negative over the entire distal half of the AAA, reaches -0.4 to $-0.5 \mathrm{~N} \mathrm{~m}^{-2}$ and the $O S I$ index a maximum of 0.8 in the region of impact of the vortex ring.

Further studies of endothelial cells subjected to the specific spatial and temporal distribution of WSS and GWSS reported here, should provide the information necessary to elucidate the possible role that these disturbed patterns of WSS may have on the aetiology and progression of AAAs. 


\section{Appendix. Calculation of the total stress}

The total stress is defined as the maximum eigenvalue of the stress tensor. It has the advantage over the shear stresses to be invariant under rotation of the coordinate axes. The stress tensor

admits three eigenvalues

$$
\sigma=\frac{1}{2} \mu\left[\nabla \boldsymbol{u}+\nabla \boldsymbol{u}^{T}\right]
$$

$$
\begin{gathered}
\tau_{1}=\frac{1}{2} \mu\left(\frac{\partial u_{r}}{\partial r}+\frac{\partial u_{y}}{\partial y}\right)+\frac{1}{2} \mu\left[\left(\frac{\partial u_{r}}{\partial r}-\frac{\partial u_{y}}{\partial y}\right)^{2}+\left(\frac{\partial u_{r}}{\partial y}+\frac{\partial u_{y}}{\partial r}\right)^{2}\right]^{0.5}, \\
\tau_{2}=\frac{1}{2} \mu\left(\frac{\partial u_{r}}{\partial r}+\frac{\partial u_{y}}{\partial y}\right)-\frac{1}{2} \mu\left[\left(\frac{\partial u_{r}}{\partial r}-\frac{\partial u_{y}}{\partial y}\right)^{2}+\left(\frac{\partial u_{r}}{\partial y}+\frac{\partial u_{y}}{\partial r}\right)^{2}\right]^{0.5}, \\
\tau_{3}=\mu \frac{u_{r}}{r},
\end{gathered}
$$

when expressed in the $(r, \theta, y)$ coordinate system. Upon a change of variables from a cylindrical to a Cartesian coordinate systems ( $y$ being in the direction of the flow and $x$ in the perpendicular direction), the total stress defined as $\tau_{1}$ takes the form

$$
\tau_{1}=\frac{1}{2} \mu\left(\frac{\partial u}{\partial x}+\frac{\partial v}{\partial y}\right)+\frac{1}{2} \mu\left[\left(\frac{\partial u}{\partial x}-\frac{\partial v}{\partial y}\right)^{2}+\left(\frac{\partial u}{\partial y}+\frac{\partial v}{\partial x}\right)^{2}\right]^{0.5} .
$$

$\tau_{1}$ can thus be calculated from the measurements of the velocity field in the plane of symmetry of the model.

\section{REFERENCES}

Asbury, C. L., Ruberti, J. W., Bluth, E. I. \& Peattie, R. A. 1995 Experimental investigation of steady flow in rigid models of abdominal aortic aneurysms. Ann. Biomed. Engng 23, 23-39.

Attinger, E. O. 1964 In Pulsatile Blood Flow: Proceedings of the First International Symposium on Pulsatile Blood Flow. (ed. E. O. Attinger). McGraw-Hill.

Attinger, E. O. 1996 In The Abdominal Aortic Aneurysm: Genetics, Pathophysiology and Molecular Biology. (ed. M. D. Tilson \& C. D. Boyd). New York Academy of Sciences.

Bengtsson, H., Sonesson, B. \& Bergqvist, D. 1996 Incidence and prevalence of abdominal aortic aneurysms, estimated by necropsy studies and population screening by ultrasound. In The Abdominal Aortic Aneurysm: Genetics, Pathophysiology and Molecular Biology (ed. M. D. Tilson \& C. D. Boyd). NY Academy of Sciences.

Best, V. A., Price, J. F. \& Fowkes, F. G. R. 2003 Persistent increase in the incidence of abdominal aortic aneurysm in Scotland, 1981-2000. Br. J. Surg. 90, 1510-1515.

Bluestein, D., Niu, L., Schoephoerster, R. T. \& Dewanjee, M. K. 1996 Steady flow in an aneurysm model: correlation between fluid dynamics and blood platelet deposition. J. Biomech. Engng 118, 280-286.

Budwig, R., Elger, D., Hooper, H. \& Slippy, J. 1993 Steady flow in abdominal aortic aneurysm models. J. Biomech. Engng 115, 418-423.

Chappell, D. C., Varner, S. E., Nerem, R. M., Medford, R. M. \& Alexander, R. W. 1998 Oscillatory shear stress stimulates adhesion molecule expression in cultured human endothelium. Circul. Res. 82, 532-539.

Chen, K. D., LI, Y. S., Kim, M., Li, S., Yuan, S., Chien, S. \& Shyy, J. Y. 1999 Mechanotransduction in response to shear stress. Roles of receptor tyrosine kinases, integrins, and Shc. J. Biol. Chem. 274, 18 393-18400. 
Cheng, C. P., Parker, D. \& TaYlor, C. A. 2002 Quantification of wall shear stress in large blood vessels using Lagrangian interpolation functions with cine phase-contrast magnetic resonance imaging. Annu. Biomed. Engng 30, 1020-1032.

Chien, S., LI, S. \& SHYY, Y. Y. 1998 Effects of mechanical forces on signal transduction and gene expression in endothelial cells. Hypertension 31, 162-169.

Darling, R. C. 1970 Ruptured arteriosclerotic abdominal aortic aneurysms. Am. J. Surg. 119, 397-401.

Davies, P. F., Mundel, T. \& Barbee, K. A. 1995 A mechanism for heterogeneous endothelial responses to flow in vivo and in vitro. J. Biomech. 28, 1553-1560.

Davies, P. F., Remuzzi, A., Gordon, E. J., Dewey Jr, C. F. \& Gibrone Jr, M. A. 1986 Turbulent fluid shear stress induces vascular endothelial turnover in vitro. Proc. Natl Acad. Sci. USA 83, 2114-2117.

DePaola, N., Gimbrone, M., Davies, P. \& Dewey, C. 1992 Vascular endothelium responds to fluid shear stress gradients. Arterioscl. Thromb. 12, 1254-1257.

Dewey, C., Bussolari, S., Gimbrone, M. \& Davies, P. 1981 The dynamic response of vascular endothelial cells to fluid shear stress. Trans. ASME K: J. Biomech. Engng 103, 177-185.

Di Martino, E., Mantero, S., Inzoli, F., Melissano, G., Astore, D., Chiesa, R. \& Fumero, R. 1998 Biomechanics of abdominal aortic aneurysm in the presence of endoluminal thrombus: experimental characterization and structure static computational analysis. Eur. J. Vasc. Endovasc. Surg. 15, 290-299.

Duncan, D. D., Bargeron, C. B., Borchardt, S. E., Deters, O. J., Gearhart, S. A., Mark, F. F. \& Friedman, M. H. 1990 The effect of compliance on wall shear in casts of a human aortic bifurcation. J. Biomech. Engng 112, 183-188.

Egelhoff, C. J., Budwig, R. S., Elger, D. F., Khraishi, T. A. \& Johansen, K. H. 1999 Model studies of the flow in abdominal aortic aneurysms during resting and exercise conditions. J. Biomech. 32, 1319-1329.

El-Khatib, F. H. \& Damiano, E. R. 2003 Linear and nonlinear analyses of pulsatile blood flow in a cylindrical tube. Biorheology 40, 503-522.

Emerson, M., Momi, S., Alberti, P. F., Page, C. \& Gresele, P. 1999 Endogenous nitric oxide acts as a natural antithrombotic agent in vivo by inhibiting platelet aggregation in the pulmonary vasculature. Thromb. Haemost. 81, 961-966.

Englund, R., Hudson, P., Hanel, K. \& Stanton, A. 1998 Expansion rates of small abdominal aortic aneurysms. Aust. N Z J. Surg. 68, 21-24.

Finol, E. A. \& Amon, C. H. 2001 Blood flow in abdominal aortic aneurysms: pulsatile flow haemodynamics. J. Biom. Engng 123, 474-484.

Finol, E. A. \& Amon, C. H. $2002 a$ Flow-induced wall shear stress in abdominal aortic aneurysms: Part I - Steady flow haemodynamics. Comput. Meth. Biomech. Biomed. Engng 5, 309-318.

Finol, E. A. \& Amon, C. H. $2002 b$ Flow-induced wall shear stress in abdominal aortic aneurysms: Part II - Pulsatile flow haemodynamics. Comput. Meth. Biomech. Biomed. Engng 5, 319-328.

Finol, E. A. \& Amon, C. H. 2003 Flow dynamics in abdominal aortic aneurysms. Acta Cient. Venez. 54, 43-49.

Fox, J. A. \& Hugh, A. E. 1966 Localization of atheroma: a theory based on boundary layer and separation. Br. Heart J. 26, 388-399.

Friedman, M. H., Hutchins, G. M., Bargeron, C. B., Deters, O. J. \& Mark, F. F. 1981 Correlation between intimal thickness and fluid shear in human arteries. Atherosclerosis 39, 425-436.

FrY, D. L. 1968 Acute vascular endothelial changes associated with increased blood velocity gradients. Circul. Res. 22, 165-197.

Fukushima, T., Matsuzawa, T. \& Homma, T. 1989 Visualization and finite element analysis of pulsatile flow in models of the abdominal aortic aneurysm. Biorheology 26, 109-130.

Gharib, M., Rambod, E. \& Shariff, K. 1998 A universal time scale for vortex ring formation. J. Fluid Mech. 360, 121-140.

Glagov, S., Zarins, C. K., Giddens, D. G. \& Ku, D. N. 1988 Haemodynamics and atherosclerosis, insights and perspectives gained from studies of human arteries. Arch. Pathol. Lab. Med. 112, $1018-1031$. 
Harter, L. P., Gross, B. H., Callen, P. W. \& Barth, R. A. 1982 Ultrasonic evaluation of abdominal aortic thrombus. J Ultrasound Med. 1, 315-318.

Hatakeyama, T., Shigematsu, H. \& Muto, T. 2001 Risk factors for rupture of abdominal aortic aneurysm based on three-dimensional study. J. Vasc. Surg. 33, 453-461.

Helpes, E. P. \& McDonald, D. A. 1954 Arterial blood flow calculated from pressure gradients. J. Physiol. 124, 30-1P.

Ku, D. N., Giddens, D. P., Zarins, C. K. \& Glagov, S. 1985 Pulsatile flow and atherosclerosis in the human carotid bifurcation. Positive correlation between plaque location and low oscillating shear stress. Arteriosclerosis. 5, 293-302.

Liu, S. Q., Tang, D., Tieche, C. \& Alkema, P. K. 2003 Pattern formation of vascular smooth muscle cells subject to nonuniform fluid shear stress: mediation by the gradient of cell density. Am. J. Physiol. Heart Circul. Physiol. 285, H1072-H1080.

Maier, S. E., Meier, D., Boesinger, P., Moser, U. T. \& Vieli, A. 1989 Human abdominal aorta: comparative measurements of blood flow with MR imaging and multigated Doppler US. Radiology 171, 487-492.

Moore, J. E., Ku, K. D., Zarins, C. K. \& Glagov, S. 1992 Pulsatile flow visualization in the abdominal aorta under differing physiologic conditions: implications for increased susceptibility to atherosclerosis. J. Biomech. Engng 114, 391-397.

Nagel, T., Resnick, N., Dewey, C. F. \& Gimbrone, M. A. 1999 Vascular endothelial cells respond to spatial gradients in fluid shear stress by enhanced activation of transcription factors. Arterioscl. Thromb. Vasc. Biol. 19, 1825-1834.

Nichols, W. W. \& O'Rourke, M. F. 1990 McDonald's blood flow in arteries: theoretic, experimental and clinical principles (ed. W. W. Nichols \& M. F. O'Rourke) Edward Arnold.

Oyre, S., Pedersen, E. M., Ringgaard, S., Boesiger, P. \& PaAske, W. P. 1997 In vivo wall shear stress measured by magnetic resonance velocity mapping in the normal human abdominal aorta. Eur. J. Vasc. Endovasc. Surg. 13, 263-271.

Peattie, R. A., Schrader, T., Bluth, E. I. \& Comstock, C. E. 1994 Development of turbulence in steady flow through models of abdominal aortic aneurysms. J. Ultrasound Med. 13, 467-472.

Pedersen, E. M., Agerbaek, M., Kristensen, I. B. \& Yoganathan, A. P. 1997 Wall shear stress and early atheroscerotic lesions in the abdominal aorta in young adults. Eur. J. Vasc. Endovasc. Surg. 13, 443-451.

Pedley, T. J. 1979 The Fluid Mechanics of Large Blood Vessels. Cambridge University Press.

Prisant, L. M. \& Mondy, J. S. 2004 Abdominal aortic aneurysms. J. Clin. Hypertens. 6, 85-89.

QIU, Y. \& TARBELL, J. M. 2000 Interaction between wall shear stress and circumferential strain affects endothelial cell biochemical production. J. Vasc. Res. 37, 147-157.

Reilly, J. M. \& Tilson, M. D. 1989 Incidence and aetiology of abdominal aortic aneurysms. Surg. Clin. North Am. 69, 705-711.

Richardson, E. G. \& TYler, W. 1929 The transverse velocity gradient near the mouths of pipes in which an alternating or continuous flow of air is established. Proc. Phys. Soc. Lond. 42, 1-15.

Salsac, A.-V., Sparks, S. R., Chomaz, J.-M. \& Lasheras, J. C. 2006 Effects of the loss of symmetry on the wall shear stresses in abdominal aortic aneurysm. J. Fluid Mech. (submitted).

Schowalter, D. G., Van Atta, C. W. \& Lasheras, J. C. 1994 A study of streamwise vortex structure in a stratified shear layer. J. Fluid Mech. 281, 247-291.

Schrader, T., Peatrie, R. A., Bluth, E. I. \& Comstock, C. E. 1992 A qualitative investigation of turbulence in flow through a model abdominal aortic aneurysm. Invest. Radiol. 27, 515-519.

Steiger, H. J., Poll, A., Liepsch, D. \& Reulen, H. J. 1987 Haemodynamic stress in lateral saccular aneurysms. An experimental study. Acta Neurochir. 86, 98-105.

Ster petti, A. V., Cucina, A., D’Angelo, L. S., Cardillo, B. \& Cavallaro, A. 1993 Shear stress modulates the proliferation rate, protein synthesis, an mitogenic activity of arterial smooth muscle cells. Surgery 113, 691-699.

Stringfellow, M. M., Lawrence, P. F. \& Stringfellow, R. G. 1987 The influence of aortaaneurysm geometry upon stress in the aneurysm wall. J. Surg. Res. 42, 425-433.

Stroud, J. S., Berger, S. A. \& SAloner, D. 2000 Influence of stenosis morphology on flow through severely stenotic vessels: implications for plaque rupture. J. Biomech. 33, 443-455.

SzILAGYI, D. E. 1982 Clinical diagnosis of intact and ruptured abdominal aortic aneurysms. In Aneurysms: Diagnosis and Treatment. (ed. J. J. Bergan \& J. S. Yao), pp. 205-215. Grune \& Stratton. 
Tardy, Y., Resnick, N., Nagel, T., Gimbrone, M. \& Dewey, C. 1997 Shear stress gradients remodel endothelial monolayer in vitro via a cell proliferation-migration-loss cycle. Arterioscl. Thromb. Vasc. Biol. 17, 3102-3106.

Taylor, C. A., Cheng, C. P., Espinosa, L. A., Tang, B. T., Parker, D. \& Herfkens, R. J. 2002 In vivo quantification of blood flow and wall shear stress in the human abdominal aorta during lower limb exercise. Ann. Biomed. Engng 30, 402-408.

TAYLOR, T. W. \& YAMAgUChI, T. 1994 Three-dimensional simulation of blood flow in an abdominal aortic aneurysm - steady and unsteady flow cases. J. Biomech. Engng 116, 89-97.

Traub, O. \& Berk, B. C. 1998 Laminar shear stress: mechanisms by which endothelial cells transduce an atheroprotective force. Arterioscl. Thromb. Vasc. Biol. 18, 677-685.

Tzima, E., Del Pozo, M. A., Kiosses, W. B., Mohamed, S. A., Li, S., Chien, S. \& Schwartz, M. A. 2002 Activation of Rac1 by shear stress in endothelial cells mediates both cytoskeletal reorganization and effects on gene expression. EMBO J. 16, 6791-6800.

Viswanath, N., RoDkiEwicz, C. M. \& ZAJAC, S. 1997 On the abdominal aortic aneurysms: pulsatile state considerations. Med. Engng Phys. 19, 343-351.

Womersley, J. R. 1954 Flow in the larger arteries and its relation to the oscillating pressure. J. Physiol. 124, 31-2P.

YIP, T. H. \& YU, S. C. M. 2001 Cyclic transition to turbulence in rigid abdominal aortic aneurysms models. Fluid Dyn. Res. 29, 81-113.

YIP, T. H. \& YU, S. C. M. 2002 Oscillatory flows in straight tubes with an axisymmetric bulge. Exp. Thermal Fluid Sci. 26, 947-961.

YU, S. C. M. 2000 Steady and pulsatile flow characteristics in abdominal aortic aneurysm models using particle image velocimetry. Intl J. Heat Fluid Flow 21, 74-83.

Yu, S. C. M., Chan, W. K., NG, B. T. H. \& ChuA, L. P. 1999 A numerical investigation on the steady and pulsatile flow characteristics in axi-symmetric abdominal aortic aneurysm models with some experimental evaluation. J. Med. Engng Tech. 23, 228-239.

YU, S. C. M. \& ZHAO, J. B. 2000 A particle image velocimetry study on the pulsatile flow characteristics in straight tubes with an asymmetric bulge. Proc. Inst. Mech. Engng C 214, 655-671.

Zhao, Y., Chen, B. P., Miao, H., Yuan, S., Li, Y. S., Hu, Y., Rocke, D. M. \& Chien, S. 2002 Improved significance test for DNA microarray data: temporal effects of shear stress on endothelial genes. Physiol. Genomics 12, 1-11. 